\title{
Null Controllability of a Four Stage and Age-Structured Population Dynamics Model
}

\author{
Amidou Traoré $\left(D,{ }^{1}\right.$ Bedr'Eddine Ainseba, ${ }^{2}$ and Oumar Traoré ${ }^{3}$ \\ ${ }^{1}$ Laboratoire LAMI, Université Joseph Ki ZERBO de Ouagadougou, 01 BP 7021 Ouaga 01, Ouagadougou, Burkina Faso \\ ${ }^{2}$ Institut de Mathématiques de Bordeaux, UMR CNRS 5251, Université de Bordeaux, 3 ter Place de la Victoire 33076, \\ Bordeaux, France \\ ${ }^{3}$ Département de Mathématiques de la Décision, Université Ougga 2, 12 BP 417 Ouaga 12, Laboratoire LAMI, \\ Université Joseph Ki ZERBO de Ouagadougou, 01 BP 7021 Ouaga 01, Ouagadougou, Burkina Faso
}

Correspondence should be addressed to Amidou Traoré; amidoutraore70@yahoo.fr

Received 16 January 2021; Revised 24 February 2021; Accepted 4 March 2021; Published 31 March 2021

Academic Editor: Valerii Obukhovskii

Copyright (C) 2021 Amidou Traoré et al. This is an open access article distributed under the Creative Commons Attribution License, which permits unrestricted use, distribution, and reproduction in any medium, provided the original work is properly cited.

This paper is devoted to study the null controllability properties of a population dynamics model with age structuring and nonlocal boundary conditions. More precisely, we consider a four-stage model with a second derivative with respect to the age variable. The null controllability is related to the extinction of eggs, larvae, and female population. Thus, we estimate a time $T$ to bring eggs, larvae, and female subpopulation density to zero. Our method combines fixed point theorem and Carleman estimate. We end this work with numerical illustrations.

\section{Introduction}

Let $u_{i}(t, a), 1 \leq i \leq 4$, be, respectively, the distribution of eggs, larvae, and female and male individuals of age $a$ at time $t$; $A_{i}, 1 \leq i \leq 4$, is the life expectancy of an $i$-stage individual and $T$ is a positive constant. We consider the following population dynamics model based on Fokker-Planck or Kolmogorov-type equations which is written as

$$
\left\{\begin{array}{l}
\partial_{t} u_{1}(t, a)+\partial_{a}\left[v_{1}(\zeta(t), a) u_{1}(t, a)\right]-k \partial_{a}^{2} u_{1}(t, a)=-\left[\mu_{1}(\zeta(t), a)+\beta_{1}(\zeta(t), a)\right] u_{1}(t, a)+m(a) w_{1}(t, a), \\
\partial_{t} u_{2}(t, a)+\partial_{a}\left[v_{2}(\zeta(t), a) u_{2}(t, a)\right]-k \partial_{a}^{2} u_{2}(t, a)=-\left[\mu_{2}(\zeta(t), a)+\beta_{2}(\zeta(t), a)\right] u_{2}(t, a)+m(a) w_{2}(t, a), \\
\partial_{t} u_{3}(t, a)+\partial_{a}\left[v_{3}(\zeta(t), a) u_{3}(t, a)\right]-k \partial_{a}^{2} u_{3}(t, a)=-\mu_{3}(\zeta(t), a) u_{3}(t, a)+m(a) w_{3}(t, a), \\
\partial_{t} u_{4}(t, a)+\partial_{a}\left[v_{4}(\zeta(t), a) u_{4}(t, a)\right]-k \partial_{a}^{2} u_{4}(t, a)=-\mu_{4}(\zeta(t), a) u_{4}(t, a),
\end{array}\right.
$$


where $(t, a) \in \Omega_{i}=(0, T) \times\left(0, A_{i}\right)$ and $w_{1}, w_{2}$, and $w_{3}$ are, respectively, the control function of eggs, larvae, and females. Here, $m(a)$ is the characteristic function of $\omega=\left(0, a^{*}\right)$ with $0<a^{*}<\min \left\{A_{1}, A_{2}, A_{3}\right\}$. To complete the system, the boundary conditions are stated as follows:

$$
\begin{cases}{\left[v_{1}(\zeta(t), a) u_{1}(t, a)-k \partial_{a} u_{1}(t, a)\right]_{a=0}} & =\int_{0}^{A_{3}} \beta_{3}(\zeta(t), a) u_{3}(t, a) \mathrm{d} a \\ {\left[v_{2}(\zeta(t), a) u_{2}(t, a)-k \partial_{a} u_{2}(t, a)\right]_{a=0}} & =\int_{0}^{A_{1}} \beta_{1}(\zeta(t), a) u_{1}(t, a) \mathrm{d} a \\ {\left[v_{3}(\zeta(t), a) u_{3}(t, a)-k \partial_{a} u_{3}(t, a)\right]_{a=0}} & =\int_{0}^{A_{2}} \sigma \beta_{2}(\zeta(t), a) u_{2}(t, a) \mathrm{d} a, \\ {\left[v_{4}(\zeta(t), a) u_{4}(t, a)-k \partial_{a} u_{4}(t, a)\right]_{a=0}} & =\int_{0}^{A_{2}}(1-\sigma) \beta_{2}(\zeta(t), a) u_{2}(t, a) \mathrm{d} a \\ u_{i}(0, a) & =u_{i}^{0}(a), a \in\left(0, A_{i}\right), \quad 1 \leq i \leq 4 \\ u_{i}\left(t, A_{i}\right) & =0, \quad t \in(0, T), 1 \leq i \leq 4\end{cases}
$$

Here, $\mu_{i}(\zeta(t), a), \beta_{i}(\zeta(t), a)$, and $v_{i}(\zeta(t), a)$ are, respectively, the $i$-stage mortality rate, the $i$-stage age specific transition functions, and the $i$-stage growth rate of age $a$ at temperature $\zeta(t)$ and at time $t$.

Finally,

$$
\int_{0}^{A_{i}} \sigma_{i} \beta_{i}(\zeta(t), a) u_{i}(t, a) \mathrm{d} a, \quad 1 \leq i \leq 4, \sigma_{1}=1, \sigma_{2}=\sigma, \sigma_{3}=1, \sigma_{4}=1-\sigma
$$

Denote, respectively, the distribution of the newborns of eggs, larvae, females, and males. The constant $\sigma$ denotes the sex ratio and $k>0$ (diffusion coefficient) corresponds to small deviations of order 2 which represent the dispersion effects of individuals during their development. Our aim is to study the null controllability of (1) and (2).

Given a fixed $T>0$, we look for controls $w_{1}, w_{2}$, and $w_{3}$ such that $u_{1}, u_{2}$, and $u_{3}$ satisfy

$$
u_{1}(T, a)=u_{2}(T, a)=u_{3}(T, a)=0 .
$$

In practice, this study applies to the dynamics cowpea pest insects (Callosobruchus maculatus). These insects live in hot areas, namely, tropical and subtropical regions [1] and cause important damage to the seeds of cowpeas and other legumes. This damage is exclusively due to the larvae. Indeed, females lay their eggs on the pods or on stocked seeds and then larvae grow by feeding on the cotyledons, see [2]. Twenty five days and a temperature varying between twentyseven and thirty-one degrees Celsius are necessary for the growth of the larvae.

The life cycle of Callosobruchus maculatus can be divided into three development stages: egg, larva + pupa (growing stages), and adult (reproduction ability). Besides, during their growth, beetle larvae transform nitrogen to toxic uric acid that accumulates into cowpea and make it unfit for consumption.

Several methods of combating these insects have been considered. Among them is the method of preservation in hermetically sealed containers, thus preventing the development of the larvae.

The method of control we are considering is to remove or eliminate individuals. This can be done with the help of pesticides. We recall that the optimal and exact control problems are widely investigated for age-structured population dynamics by many researchers. Most of these studies are focused on optimal control problems [3-5] and the references therein. One can also refer to $[6,7]$. Lebeau and Robbiano establish in [8] the null controllability of the linear heat equation. Next, the exact controllability for age dependent linear and nonlinear single-species population models with spatial diffusion was investigated by Aïnsebaet al. [9, 10]. Barbu et al. also considered the exact controllability of the linear Lotka-McKendrick model without spatial structure by establishing an observability inequality for the backward adjoint system [11]. Later on, He and Ainseba investigate the exact null controllability of a stage and age-structured population dynamics system in [12] and the exact null controllability of the Lobesia Botrana model with spatial diffusion in [13].

Moreover, in [14], the authors investigated a semilinear problem: the null controllability of the heat equation with a Fourier boundary condition over the entire boundary. In our work, the Fourier condition contains a nonlocal term and is set only on a part of the domain. This induces additional difficulties in establishing the Carleman inequality.

In [15], Hegoburu and Anita study in a very practical way the null controllability of a nonlinear model of 
population dynamics by means of a feedback control. This interesting result cannot be applied easily in our case since we study a four-stage model via three controls. More precisely, in our case, we control only three stages. So, it seems difficult here to compute explicitly the feedback control as it was done in [15].

The fundamental difference with [13] lies in the fact that our model combines a transport effect with the presence of a first-order derivation with respect to the age variable and a diffusion along the same variable. Thus, the inequalities guaranteeing the null controllability obtained in [13] become inoperative in our case.

As far as we know, there are no results dealing with the null controllability for an age and stage-dependent system involving a second derivative with respect to the age variable. The second derivative with respect to the physiological age variable corresponds to small deviations of order two and represents the dispersion effects of individuals during their development due to, for example, the temperature, the humidity, and the quality of food. This article is structured as follows. In Section 2, the assumptions and the main result are stated. We study the null controllability of some associated auxiliary model in Section 3. Section 4 is devoted to the proof of the main result, and some numerical illustrations are given in Section 5.

\section{Assumptions and Main Result}

For the sequel, we make the following assumptions on the demographic parameters:

(A1) The transition functions $\beta_{k}(\zeta(),$.$) are positive$ and $\beta_{k} \in L^{\infty}\left((0, T) \times\left(0, A_{k}\right)\right), 1 \leq k \leq 3$.

(A2) The mortality functions $\mu_{k}(\zeta(),$.$) are positive and$ $\mu_{k} \in L^{\infty}\left((0, T) \times\left(0, A_{k}\right)\right), 1 \leq k \leq 4$.

(A3) The initial conditions $u_{k}^{0}(a) \geq 0$ a.e. $a \in$ $(0, A), 1 \leq k \leq 4$.

(A4) The growth functions $v_{i}(\zeta(),),. 1 \leq i \leq 4$ are positive and bounded, that is,

$0<v_{i}^{\min }<v_{i}(\zeta(t), a)<v_{i}^{\max }, \quad \forall(t, a) \in(0, T) \times\left(0, A_{i}\right)$.

In addition, for all $t \in[0, T], v_{i}(\zeta(t),.) \in C^{1}([0, A])$, and there is a positive constant $C_{i}$ such that

$$
\left\|\partial_{a} v_{i}(\zeta(t), a)\right\|_{\infty} \leq C_{i}, \quad \forall(t, a) \in(0, T) \times\left(0, A_{i}\right) .
$$

(A5) The temperature function $\zeta$ (.) is measurable.

These assumptions are biologically meaningful, see [3, 16-18].

Setting $\widehat{u}_{i}=e^{-\lambda t} u_{i}, 1 \leq i \leq 4$, the vector $\left(\widehat{u}_{1}, \widehat{u}_{2}, \widehat{u}_{3}, \widehat{u}_{4}\right)$ verifies the system:

$$
\left\{\begin{array}{l}
\partial_{t} \widehat{u}_{1}+\partial_{a}\left(v_{1} \widehat{u}_{1}\right)-k \partial_{a}^{2} \widehat{u}_{1}=-\left(\mu_{1}+\beta_{1}+\lambda\right) \widehat{u}_{1}+m(a) \widehat{w}_{1}, \\
\partial_{t} \widehat{u}_{2}+\partial_{a}\left(v_{2} \widehat{u}_{2}\right)-k \partial_{a}^{2} \widehat{u}_{2}=-\left(\mu_{2}+\beta_{2}+\lambda\right) \widehat{u}_{2}+m(a) \widehat{w}_{2}, \\
\partial_{t} \widehat{u}_{3}+\partial_{a}\left(v_{3} \widehat{u}_{3}\right)-k \partial_{a}^{2} \widehat{u}_{3}=-\left(\mu_{3}+\lambda\right) \widehat{u}_{3}+m(a) \widehat{w}_{3}, \\
\partial_{t} \widehat{u}_{4}+\partial_{a}\left(v_{4} \widehat{u}_{4}\right)-k \partial_{a}^{2} \widehat{u}_{4}=-\left(\mu_{4}+\lambda\right) \widehat{u}_{4}, \\
{\left[v_{2}(\zeta(t), a) \widehat{u}_{2}(t, a)-k \partial_{a} \widehat{u}_{2}(t, a)\right]_{a=0}=\int_{0}^{A_{1}} \beta_{1}(\zeta(t), a) \widehat{u}_{1}(t, a) \mathrm{d} a,} \\
{\left[v_{3}(\zeta(t), a) \widehat{u}_{3}(t, a)-k \partial_{a} \widehat{u}_{3}(t, a)\right]_{a=0}=\int_{0}^{A_{2}} \sigma \beta_{2}(\zeta(t), a) \widehat{u}_{2}(t, a) \mathrm{d} a,} \\
{\left[v_{4}(\zeta(t), a) \widehat{u}_{4}(t, a)-k \partial_{a} \widehat{u}_{4}(t, a)\right]_{a=0}=\int_{0}^{A_{2}}(1-\sigma) \beta_{2}(\zeta(t), a) \widehat{u}_{2}(t, a) \mathrm{d} a,} \\
\widehat{u}_{i}(0, a)=\widehat{u}^{0}(a), a \in\left(0, A_{i}\right) \text { and } \widehat{u}_{i}\left(t, A_{i}\right)=0, t \in(0, T), \quad 1 \leq i \leq 4 .
\end{array}\right.
$$

Now, we consider the following auxiliary system: 


$$
\begin{aligned}
& \left\{\begin{array}{l}
\partial_{t} \widehat{u}_{1}+\partial_{a}\left(v_{1} \widehat{u}_{1}\right)-k \partial_{a}^{2} \widehat{u}_{1}=-\left(\mu_{1}+\beta_{1}+\lambda\right) \widehat{u}_{1}+m(a) \widehat{w}_{1}, \\
\partial_{t} \widehat{u}_{2}+\partial_{a}\left(v_{2} \widehat{u}_{2}\right)-k \partial_{a}^{2} \widehat{u}_{2}=-\left(\mu_{2}+\beta_{2}+\lambda\right) \widehat{u}_{2}+m(a) \widehat{w}_{2}, \\
\partial_{t} \widehat{u}_{3}+\partial_{a}\left(v_{3} \widehat{u}_{3}\right)-k \partial_{a}^{2} \widehat{u}_{3}=-\left(\mu_{3}+\lambda\right) \widehat{u}_{3}+m(a) \widehat{w}_{3}, \\
\partial_{t} \widehat{u}_{4}+\partial_{a}\left(v_{4} \widehat{u}_{4}\right)-k \partial_{a}^{2} \widehat{u}_{4}=-\left(\mu_{4}+\lambda\right) \widehat{u}_{4},
\end{array}\right. \\
& \left\{\begin{array}{l}
{\left[v_{1}(\zeta(t), a) \widehat{u}_{1}(t, a)-k \partial_{a} \widehat{u}_{1}(t, a)\right]_{a=0}=\int_{0}^{A_{3}} \beta_{3}(\zeta(t), a) \phi_{3}(t, a) \mathrm{d} a,} \\
{\left[v_{2}(\zeta(t), a) \widehat{u}_{2}(t, a)-k \partial_{a} \widehat{u}_{2}(t, a)\right]_{a=0}=\int_{0}^{A_{1}} \beta_{1}(\zeta(t), a) \phi_{1}(t, a) \mathrm{d} a,} \\
{\left[v_{3}(\zeta(t), a) \widehat{u}_{3}(t, a)-k \partial_{a} \widehat{u}_{3}(t, a)\right]_{a=0}=\int_{0}^{A_{2}} \sigma \beta_{2}(\zeta(t), a) \phi_{2}(t, a) \mathrm{d} a,} \\
{\left[v_{4}(\zeta(t), a) \widehat{u}_{4}(t, a)-k \partial_{a} \widehat{u}_{4}(t, a)\right]_{a=0}=\int_{0}^{A_{2}}(1-\sigma) \beta_{2}(\zeta(t), a) \phi_{2}(t, a) \mathrm{d} a,} \\
\widehat{u}_{i}(0, a)=\widehat{u}^{0}(a), \quad a \in\left(0, A_{i}\right) \text { and } \widehat{u}_{i}\left(t, A_{i}\right)=0, t \in(0, T), 1 \leq i \leq 4 .
\end{array}\right.
\end{aligned}
$$

For the existence of the solutions to problems similar to equations (8) and (9), we refer, for instance, to [19] and the references therein.

Let $A=\max \left\{A_{1}, A_{2}, A_{3}, A_{4}\right\}$, we can state the main result.

Theorem 1. Assume that $(A 1)-(A 5)$ hold. Let $A>0$ and $T>0$ be given. Then, for all $\left(u_{1}^{0}, u_{2}^{0}, u_{3}^{0}\right) \in\left(L^{2}(0, A)\right)^{3}$, there exist a control $w=\left(w_{1}, w_{2}, w_{3}\right) \in\left(L^{2}((0, T) \times \omega)\right)^{3}$ such that the associated solution of equations (1) and (2) verify (4).

\section{Null Controllability of an Auxiliary System}

This section is devoted to the null controllability of the following auxiliary system obtained from equations (1) and (2):

$$
\begin{aligned}
& \left\{\begin{array}{l}
\partial_{t} u_{1}(t, a)+\partial_{a}\left[v_{1}(\zeta(t), a) u_{1}(t, a)\right]-k \partial_{a}^{2} u_{1}(t, a)=-\left[\mu_{1}(\zeta(t), a)+\beta_{1}(\zeta(t), a)\right] u_{1}(t, a)+m(a) w_{1}(t, a) \\
\partial_{t} u_{2}(t, a)+\partial_{a}\left[v_{2}(\zeta(t), a) u_{2}(t, a)\right]-k \partial_{a}^{2} u_{2}(t, a)=-\left[\mu_{2}(\zeta(t), a)+\beta_{2}(\zeta(t), a)\right] u_{2}(t, a)+m(a) w_{2}(t, a) \\
\partial_{t} u_{3}(t, a)+\partial_{a}\left[v_{3}(\zeta(t), a) u_{3}(t, a)\right]-k \partial_{a}^{2} u_{3}(t, a)=-\mu_{3}(\zeta(t), a) u_{3}(t, a)+m(a) w_{3}(t, a) \\
\partial_{t} u_{4}(t, a)+\partial_{a}\left[v_{4}(\zeta(t), a) u_{4}(t, a)\right]-k \partial_{a}^{2} u_{4}(t, a)=-\mu_{4}(\zeta(t), a) u_{4}(t, a)
\end{array}\right. \\
& \begin{cases}{\left[v_{1}(\zeta(t), a) u_{1}(t, a)-k \partial_{a} u_{1}(t, a)\right]_{a=0}} & =h_{1}(t), \\
{\left[v_{2}(\zeta(t), a) u_{2}(t, a)-k \partial_{a} u_{2}(t, a)\right]_{a=0}} & =h_{2}(t), \\
{\left[v_{3}(\zeta(t), a) u_{3}(t, a)-k \partial_{a} u_{3}(t, a)\right]_{a=0}} & =h_{3}(t), \\
{\left[v_{4}(\zeta(t), a) u_{4}(t, a)-k \partial_{a} u_{4}(t, a)\right]_{a=0}} & =h_{4}(t), \\
u_{i}(0, a) & =u_{i}^{0}(a), \quad a \in(0, A), 1 \leq i \leq 4, \\
u_{i}(t, a) & =0, \quad t \in(0, T), 1 \leq i \leq 4 .\end{cases}
\end{aligned}
$$

Here, $h_{i}=\int_{0}^{A} \sigma_{i} \beta_{j}(\zeta(t), a) \phi_{j}(t, a) \mathrm{d} a,\left(i, \sigma_{i}, j\right) \in\{(1,1,3$ )$,(2,1,1),(3, \sigma, 2),(4,1-\sigma, 2)\}$. We first established a
Carleman-type inequality for the following adjoint system of equations (10) and (11): 


$$
\left\{\begin{array}{l}
-\partial_{t} q_{1}-\partial_{a}\left(v_{1} q_{1}\right)-k \partial_{a}^{2} q_{1}+\left(\mu_{1}+\beta_{1}+\partial_{a} v_{1}\right) q_{1}=0, \\
-\partial_{t} q_{2}-\partial_{a}\left(v_{2} q_{2}\right)-k \partial_{a}^{2} q_{2}+\left(\mu_{2}+\beta_{2}+\partial_{a} v_{2}\right) q_{2}=0, \\
-\partial_{t} q_{3}-\partial_{a}\left(v_{3} q_{3}\right)-k \partial_{a}^{2} q_{3}+\left(\mu_{3}+\partial_{a} v_{3}\right) q_{3}=0, \\
-\partial_{t} q_{4}-\partial_{a}\left(v_{4} q_{4}\right)-k \partial_{a}^{2} q_{4}+\left(\mu_{4}+\partial_{a} v_{4}\right) q_{4}=0,
\end{array}\right.
$$

with $(t, a) \in Q$ and the following boundary conditions

$$
\left\{\begin{array}{l}
q_{i}(t, A)=\partial_{a} q_{i}(t, 0)=0, \quad t \in(0, T), 1 \leq i \leq 4 ; \\
q_{i}(T, a)=-\frac{u_{i}(T, a)}{\varepsilon}, \quad a \in(0, A), 1 \leq i \leq 3 ; \\
q_{4}(T, a)=0, \quad a \in(0, A) .
\end{array}\right.
$$

The system equations (12) and (13) admit a unique solution.

Let $\omega_{0} \subset \subset \omega$ be a nonempty bounded subset of $\Omega=(0, A)$, and $\psi \in C^{2}(\bar{\Omega})$ satisfying (see Lemma 1.1 in [20])

$$
\begin{aligned}
\psi(a)>0 ; & \text { for any } a \in \Omega, \psi(a)=0, \\
& \text { for any } a \in \partial \Omega,|\nabla \psi(a)|>0, \\
& \text { for any } a \in \overline{\Omega \backslash \omega_{0}} .
\end{aligned}
$$

Now, we define the following weight functions, which are constructed using the function $\psi$ (see [21] or [20]):

$$
\left\{\begin{array}{l}
\varphi(t, a)=\frac{e^{\lambda \psi(a)}}{t(T-t)}, \alpha(t, a)=\frac{-e^{2 \lambda\|\psi\|_{C(\bar{\Omega})}}+e^{\lambda \psi(a)}}{t(T-t)}, \\
\widetilde{\varphi}(t, a)=\frac{e^{-\lambda \psi(a)}}{t(T-t)}, \widetilde{\alpha}(t, a)=\frac{-e^{2 \lambda\|\psi\|_{C(\bar{\Omega})}}+e^{-\lambda \psi(a)}}{t(T-t)},
\end{array}\right.
$$

where $\lambda$ is an appropriate positive constant.

Remark 1. Using the definition of $\varphi, \widetilde{\varphi}, \alpha$, and $\widetilde{\alpha}$, we deduce that there is a positive constant $c$ such that

(1) $\partial_{a} \varphi=\lambda \partial_{a} \psi \varphi, \partial_{a} \widetilde{\varphi}=-\lambda \partial_{a} \psi \widetilde{\varphi}, \partial_{a} \alpha=\lambda \partial_{a} \psi \varphi$, and $\partial_{a} \widetilde{\alpha}$ $=-\lambda \partial_{a} \psi \widetilde{\varphi}$

(2) $\left|\partial_{t} \varphi\right| \leq c \varphi^{2},\left|\partial_{t} \widetilde{\varphi}\right| \leq c \widetilde{\varphi}^{2},\left|\partial_{t} \alpha\right| \leq c \varphi^{2},\left|\partial_{t} \widetilde{\alpha}\right| \leq c \widetilde{\varphi}^{2},\left|\partial_{t}^{2} \varphi\right|$ $\leq c \varphi^{3},\left|\partial_{t}^{2} \widetilde{\varphi}\right| \leq c \widetilde{\varphi}^{3},\left|\partial_{t}^{2} \alpha\right| \leq c \varphi^{3},\left|\partial_{t}^{2} \widetilde{\alpha}\right| \leq c \widetilde{\varphi}^{3},\left|\partial_{t} \partial_{a} \alpha\right| \leq$ $c \varphi^{2}$, and $\left|\partial_{t} \partial_{a} \widetilde{\alpha}\right| \leq c \widetilde{\varphi}^{2}$

Lemma 1. Let $\varphi, \alpha, \widetilde{\varphi}$, and $\widetilde{\alpha}$ be defined as in equation (15). Then, there exists a number $\hat{\lambda}>1$ such that, for any $\lambda>\hat{\lambda}$, there exists a number $s_{0}(\lambda)$ such that, for every $s \geq s_{0}(\lambda)$, we have the following inequalities:

$$
\begin{aligned}
& \int_{Q} {\left[\frac{1}{s \varphi}\left(\left|\partial_{t} q_{i}\right|^{2}+\left|\partial_{a}^{2} q_{i}\right|^{2}\right)+s \varphi\left|\partial_{a} q_{i}\right|^{2}+s^{3} \varphi^{3} q_{i}^{2}\right]\left(e^{2 s \alpha}+e^{2 s \widetilde{\alpha}}\right) \mathrm{d} t \mathrm{~d} x \leq C } \\
& \cdot \int_{[0, T] \times \omega} s^{3} \varphi^{3} q_{i}^{2}\left(e^{2 s \alpha}+e^{2 \widetilde{\alpha}}\right) \mathrm{d} t \mathrm{~d} a
\end{aligned}
$$

for all $q_{i} \in L^{2}((0, T) \times(0, A))$ solution of (12) and (13), $i=1,2,3$, and

$$
\begin{aligned}
& \int_{Q} \varphi^{3}\left(q_{1}^{2}+q_{2}^{2}+q_{3}^{2}\right)\left(e^{2 s \alpha}+e^{2 s \tilde{\alpha}}\right) \mathrm{d} t \mathrm{~d} a \leq C \\
& \cdot \int_{[0, T] \times \omega} \varphi^{3}\left(q_{1}^{2}+q_{2}^{2}+q_{3}^{2}\right)\left(e^{2 s \alpha}+e^{2 s \tilde{\alpha}}\right) \mathrm{d} t \mathrm{~d} a .
\end{aligned}
$$

Remark 2. Here, we have to control the boundary conditions which is not the case studied in [20].

Proof of Lemma 1. Let us consider the operator

$$
\widehat{L} q_{i}=-\partial_{t} q_{i}-k \partial_{a}^{2} q_{i}, \quad 1 \leq i \leq 3 .
$$

We set

$$
\tilde{g}_{i}(t, a)=-\left(\mu_{i}+\beta_{i}\right) q_{i}+v_{i} \partial_{a} q_{i}, \quad\left(\beta_{3}=\beta_{4}=0\right), 1 \leq i \leq 3 .
$$

Let $w_{i}=e^{s \alpha} q_{i}$ and $\widetilde{w}_{i}=e^{\tilde{s \alpha}} q_{i},(1 \leq i \leq 3)$, where $s$ is a positive constant. Put $\Sigma_{0}=[0, T] \times\{A\}, \Sigma_{1}=[0, T] \times\{0\}$, and $\Sigma=\Sigma_{0} \cup \Sigma_{1}$.

From (15), we have

$$
w_{i}(T, a)=\widetilde{w}_{i}(T, a)=w_{i}(0, a)=\widetilde{w}_{i}(0, a)=0 \text { on }[0, A] .
$$

Let us define the operators $P$ and $\widetilde{P}$ as follows: $P w_{i}=e^{s \alpha} \widehat{L} e^{-s \alpha} w_{i}$ and $\widetilde{P} w_{i}=e^{s \tilde{\alpha}} \widehat{L} e^{-s \tilde{\alpha}} w_{i}$.

It follows from equations (12), (18), and (19) that

$$
\begin{array}{ll}
P w_{i}=e^{s \alpha} \widehat{L} e^{-s \alpha} w_{i}=e^{s \alpha} \widetilde{g}_{i}, & \text { on } Q, \\
\widetilde{P} \widetilde{w}_{i}=e^{\widetilde{s \alpha}} \widehat{L} e^{-s \alpha} \widetilde{w}_{i}=e^{\widetilde{s \alpha}} \widetilde{g}_{i}, & \text { on } Q .
\end{array}
$$

Explicitly, we have 


$$
\begin{aligned}
P w_{i}= & -\partial_{t} w_{i}-k \partial_{a}^{2} w_{i}+s \partial_{t} \alpha w_{i}+2 k s \lambda \partial_{a} \psi \varphi \partial_{a} w_{i}+k s \lambda \partial_{a}^{2} \psi \varphi w_{i}+k s \lambda^{2}\left(\partial_{a} \psi\right)^{2} \varphi w_{i} \\
& -k s^{2} \lambda^{2}\left(\partial_{a} \psi\right)^{2} \varphi^{2} w_{i}, \\
\widetilde{P} \widetilde{w}_{i}= & -\partial_{t} \widetilde{w}_{i}-k \partial_{a}^{2} \widetilde{w}_{i}+s \partial_{t} \widetilde{\alpha} \widetilde{w}_{i}-2 k s \lambda \partial_{a} \psi \widetilde{\varphi} \partial_{a} \widetilde{w}_{i}-k s \lambda \partial_{a}^{2} \psi \widetilde{\varphi} \widetilde{w}_{i}+k s \lambda^{2}\left(\partial_{a} \psi\right)^{2} \widetilde{\varphi} \widetilde{w}_{i} \\
& -k s^{2} \lambda^{2}\left(\partial_{a} \psi\right)^{2} \varphi^{2} \widetilde{w}_{i} .
\end{aligned}
$$

We also consider the operators $L_{1}, L_{2}, \widetilde{L}_{1}$, and $\widetilde{L}_{2}$ defined as follows:

$$
\left\{\begin{array}{l}
L_{1} w_{i}=-k \partial_{a}^{2} w_{i}-k s^{2} \lambda^{2}\left(\partial_{a} \psi\right)^{2} \varphi^{2} w_{i}+s \partial_{t} \alpha w_{i}, \\
L_{2} w_{i}=-\partial_{t} w_{i}+2 k s \lambda \partial_{a} \psi \varphi \partial_{a} w_{i}+2 k s \lambda^{2}\left(\partial_{a} \psi\right)^{2} \varphi w_{i}, \\
\widetilde{L}_{1} \widetilde{w}_{i}=-k \partial_{a}^{2} \widetilde{w}_{i}-k s^{2} \lambda^{2}\left(\partial_{a} \psi\right)^{2} \widetilde{\varphi}^{2} \widetilde{w}_{i}+s \partial_{t} \widetilde{\alpha} \widetilde{w}_{i}, \\
\widetilde{L}_{2} \widetilde{w}_{i}=-\partial_{t} \widetilde{w}_{i}-2 k s \lambda \partial_{a} \psi \widetilde{\varphi} \partial_{a} \widetilde{w}_{i}+2 k s \lambda^{2}\left(\partial_{a} \psi\right)^{2} \widetilde{\varphi} \widetilde{w}_{i} .
\end{array}\right.
$$

Using equations (19), (22), and (24), we obtain

$$
\begin{cases}L_{1} w_{i}+L_{2} w_{i}=f_{s}, & \text { in } Q, \\ \widetilde{L}_{1} \widetilde{w}_{i}+\widetilde{L}_{2} \widetilde{w}_{i}=\widetilde{f}_{s}, & \text { in } Q,\end{cases}
$$

where

$$
\left\{\begin{array}{l}
f_{s}=\widetilde{g}_{i} e^{s \alpha}-k s \lambda \varphi \partial_{a}^{2} \psi w_{i}+k s \lambda^{2} \varphi\left(\partial_{a} \psi\right)^{2} w_{i}, \\
\widetilde{f}_{s}=\widetilde{g}_{i} e^{s \tilde{\alpha}}+k s \lambda \widetilde{\varphi} \partial_{a}^{2} \psi \widetilde{w}_{i}+k s \lambda^{2} \widetilde{\varphi}\left(\partial_{a} \psi\right)^{2} \widetilde{w}_{i} .
\end{array}\right.
$$

Taking the $L_{2}$-norm of (25), we obtain

$$
\left\{\begin{array}{l}
\left\|f_{s}\right\|_{L^{2}(Q)}^{2}=\left\|L_{1} w_{i}\right\|_{L^{2}(Q)}^{2}+\left\|L_{2} w_{i}\right\|_{L^{2}(Q)}^{2}+2\left(L_{1} w_{i}, L_{2} w_{i}\right)_{L^{2}(Q)}, \\
\left\|\widetilde{f}_{s}\right\|_{L^{2}(Q)}^{2}=\left\|\widetilde{L}_{1} \widetilde{w}_{i}\right\|_{L^{2}(Q)}^{2}+\left\|\widetilde{L}_{2} \widetilde{w}_{i}\right\|_{L^{2}(Q)}^{2}+2\left(\widetilde{L}_{1} \widetilde{w}_{i}, \widetilde{L}_{2} \widetilde{w}_{i}\right)_{L^{2}(Q)}
\end{array}\right.
$$

By using (24), we have the following equalities:

$$
\begin{aligned}
2\left(L_{1} w_{i}, L_{2} w_{i}\right)_{L^{2}(Q)}= & 2 k^{2} s^{3} \lambda^{4} \int_{Q}\left(w_{i}\right)^{2} \varphi^{3}\left(\partial_{a} \psi\right)^{4} \mathrm{~d} t \mathrm{~d} a+6 k^{2} s \lambda^{2} \int_{Q}\left(\partial_{a} w_{i}\right)^{2} \varphi\left(\partial_{a} \psi\right)^{2} \mathrm{~d} t \mathrm{~d} a \\
& -2 k s \lambda \int_{0}^{T}\left[w_{i} \partial_{t} w_{i} \varphi\left(\partial_{a} \psi\right)\right]_{a=0} \mathrm{~d} t+4 k^{2} s^{2} \lambda^{3} \int_{0}^{T}\left[\left(w_{i}\right)^{2} \varphi^{2}\left(\partial_{a} \psi\right)^{3}\right]_{a=0} \mathrm{~d} t \\
& +2 k^{2} s^{3} \lambda^{3} \int_{0}^{T}\left[\left(w_{i}\right)^{2} \varphi^{3}\left(\partial_{a} \psi\right)^{3}\right]_{a=0} \mathrm{~d} t-2 k^{2} s \lambda \int_{0}^{T}\left[\left(\partial_{a} w_{i}\right)^{2} \varphi\left(\partial_{a} \psi\right)\right]_{0}^{A} \mathrm{~d} t \\
& -2 k s^{2} \lambda \int_{0}^{T}\left[\left(w_{i}\right)^{2} \varphi\left(\partial_{a} \psi\right) \partial_{t} \alpha\right]_{a=0} \mathrm{~d} t+X_{1}, \\
2\left(\widetilde{L}_{1} \widetilde{w}_{i}, \widetilde{L}_{2} \widetilde{w}_{i}\right)_{L^{2}(Q)}= & 2 k^{2} s^{3} \lambda^{4} \int_{Q}\left(\widetilde{w}_{i}\right)^{2} \widetilde{\varphi}^{3}\left(\partial_{a} \psi\right)^{4} \mathrm{~d} t \mathrm{~d} a+6 k^{2} s \lambda^{2} \int_{Q}\left(\partial_{a} \widetilde{w}_{i}\right)^{2} \widetilde{\varphi}\left(\partial_{a} \psi\right)^{2} \mathrm{~d} t \mathrm{~d} a \\
& +2 k s \lambda \int_{0}^{T}\left[\widetilde{w}_{i} \partial_{t} \widetilde{w}_{i} \widetilde{\varphi}\left(\partial_{a} \psi\right)\right]_{a=0} \mathrm{~d} t-4 k^{2} s^{2} \lambda^{3} \int_{0}^{T}\left[\left(\widetilde{w}_{i}\right)^{2} \widetilde{\varphi}^{2}\left(\partial_{a} \psi\right)^{3}\right]_{a=0} \mathrm{~d} t \\
& -2 k^{2} s^{3} \lambda^{3} \int_{0}^{T}\left[\left(\widetilde{w}_{i}\right)^{2} \widetilde{\varphi}^{3}\left(\partial_{a} \psi\right)^{3}\right]_{a=0} \mathrm{~d} t+2 k^{2} s \lambda \int_{0}^{T}\left[\left(\partial_{a} \widetilde{w}_{i}\right)^{2} \widetilde{\varphi}\left(\partial_{a} \psi\right)\right]_{0}^{A} \mathrm{~d} t \\
& +2 k s^{2} \lambda \int_{0}^{T}\left[\left(\widetilde{w}_{i}\right)^{2} \widetilde{\varphi}\left(\partial_{a} \psi\right) \partial_{t} \widetilde{\alpha}\right]_{a=0} \mathrm{~d} t+X_{2},
\end{aligned}
$$

with

$$
\begin{aligned}
& \left|X_{1}\right| \leq c_{1} \int_{Q}\left[\left(s^{3} \lambda^{3} \varphi^{3}+s^{2} \lambda^{4} \varphi^{3}\right)\left(w_{i}\right)^{2}+(s \lambda \varphi+1)\left|\partial_{a} w_{i}\right|^{2}\right] \mathrm{d} t \mathrm{~d} a, \quad \forall s \geq 1, \lambda \geq 1, \\
& \left|X_{2}\right| \leq c_{2} \int_{Q}\left[\left(s^{3} \lambda^{3} \widetilde{\varphi}^{3}+s^{2} \lambda^{4} \widetilde{\varphi}^{3}\right)\left(\widetilde{w}_{i}\right)^{2}+(s \lambda \widetilde{\varphi}+1)\left|\partial_{a} \widetilde{w}_{i}\right|^{2}\right] \mathrm{d} t \mathrm{~d} a, \quad \forall s \geq 1, \lambda \geq 1 .
\end{aligned}
$$


Considering equations (27)-(29) and using the fact that $\psi(a)=0$ on $\partial \Omega$, it follows that

$$
\begin{aligned}
\left\|f_{s}\right\|_{L^{2}(Q)}^{2}+\left\|\widetilde{f}_{s}\right\|_{L^{2}(Q)}^{2}= & \left\|L_{1} w_{i}\right\|_{L^{2}(Q)}^{2}+\left\|L_{2} w_{i}\right\|_{L^{2}(Q)}^{2}+\left\|\widetilde{L}_{1} \widetilde{w}_{i}\right\|_{L^{2}(Q)}^{2}+\left\|\widetilde{L}_{2} \widetilde{w}_{i}\right\|_{L^{2}(Q)}^{2} \\
& +2 X_{1}+2 X_{2}+2 k^{2} s^{3} \lambda^{4} \int_{Q}\left[\left(w_{i}\right)^{2} \varphi^{3}\left(\partial_{a} \psi\right)^{4}+\left(\widetilde{w}_{i}\right)^{2} \widetilde{\varphi}^{3}\left(\partial_{a} \psi\right)^{4}\right] \mathrm{d} t \mathrm{~d} a \\
& +6 k^{2} s \lambda^{2} \int_{Q}\left[\left(\partial_{a} w_{i}\right)^{2} \varphi\left(\partial_{a} \psi\right)^{2}+\left(\partial_{a} \widetilde{w}_{i}\right)^{2} \widetilde{\varphi}\left(\partial_{a} \psi\right)^{2}\right] \mathrm{d} t \mathrm{~d} a,
\end{aligned}
$$

and we deduce from (32)

$$
\begin{aligned}
\left\|f_{s}\right\|_{L^{2}(Q)}^{2}+\left\|\tilde{f}_{s}\right\|_{L^{2}(Q)}^{2} \geq & \left\|L_{1} w_{i}\right\|_{L^{2}(Q)}^{2}+\left\|L_{2} w_{i}\right\|_{L^{2}(Q)}^{2}+\left\|\widetilde{L}_{1} \widetilde{w}_{i}\right\|_{L^{2}(Q)}^{2}+\left\|\widetilde{L}_{2} \widetilde{w}_{i}\right\|_{L^{2}(Q)}^{2} \\
& +X_{1}+X_{2}+2 k^{2} s^{3} \lambda^{4} \int_{Q}\left[\left(w_{i}\right)^{2} \varphi^{3}\left(\partial_{a} \psi\right)^{4}+\left(\widetilde{w}_{i}\right)^{2} \widetilde{\varphi}^{3}\left(\partial_{a} \psi\right)^{4}\right] \mathrm{d} t \mathrm{~d} a \\
& +2 k^{2} s \lambda^{2} \int_{Q}\left[\left(\partial_{a} w_{i}\right)^{2} \varphi\left(\partial_{a} \psi\right)^{2}+\left(\partial_{a} \widetilde{w}_{i}\right)^{2} \widetilde{\varphi}\left(\partial_{a} \psi\right)^{2}\right] \mathrm{d} t \mathrm{~d} a .
\end{aligned}
$$

Note that there exists $\delta>0$ such that

$$
\left|\partial_{a} \psi(a)\right|>\delta>0, \quad \forall a \in \overline{\Omega \backslash \omega_{0}} .
$$

After these calculations, we can now use the same arguments developed in [21] or [20] to get (16).

By summing with respect to $i$, one deduces easily inequality (17). We can now establish the observability inequality of the system equations (12) and (13) and then the null controllability result of the system equations (10) and (11). We start by proving our observability inequality by means of Carleman estimate.

Lemma 2. We have the following inequality:

$$
\begin{aligned}
& \int_{0}^{A} \sum_{i=1}^{3}\left|q_{i}(0, a)\right|^{2} \mathrm{~d} a+\int_{0}^{T} \sum_{i=1}^{3}\left|q_{i}(0, a)\right|^{2} \mathrm{~d} t \\
& \leq K \int_{[0, T] \times \omega} \sum_{i=1}^{3}\left|q_{i}\right|^{2} \mathrm{~d} t \mathrm{~d} a,
\end{aligned}
$$

for all solution, $q=\left(q_{1}, q_{2}, q_{3}\right) \in\left(L^{2}((0, T) \times(0, A))\right)^{3}$ of equations (12) and (13).

Let $\lambda$ be a positive constant and $\hat{q}_{i}(t, a)=$ $e^{\lambda t} q_{i}(t, a), 1 \leq i \leq 4$. Then, equations (12) and (13) become

$$
\left\{\begin{array}{l}
-\partial_{t} \widehat{q}_{1}-\partial_{a}\left(v_{1} \widehat{q}_{1}\right)-k \partial_{a}^{2} \widehat{q}_{1}+\left(\lambda+\mu_{1}+\beta_{1}+\partial_{a} v_{1}\right) \widehat{q}_{1}=0 \\
-\partial_{t} \widehat{q}_{2}-\partial_{a}\left(v_{2} \widehat{q}_{2}\right)-k \partial_{a}^{2} \widehat{q}_{2}+\left(\lambda+\mu_{2}+\beta_{2}+\partial_{a} v_{2}\right) \widehat{q}_{2}=0 \\
-\partial_{t} \widehat{q}_{3}-\partial_{a}\left(v_{3} \widehat{q}_{3}\right)-k \partial_{a}^{2} \widehat{q}_{3}+\left(\lambda+\mu_{3}+\partial_{a} v_{3}\right) \widehat{q}_{3}=0 \\
-\partial_{t} \widehat{q}_{4}-\partial_{a}\left(v_{4} \widehat{q}_{4}\right)-k \partial_{a}^{2} \widehat{q}_{4}+\left(\lambda+\mu_{4}+\partial_{a} v_{4}\right) \widehat{q}_{4}=0
\end{array}\right.
$$

$$
\left\{\begin{array}{l}
\widehat{q}_{i}(t, A)=\partial_{a} \widehat{q}_{i}(t, 0)=0, \quad t \in(0, T), 1 \leq i \leq 4 ; \\
\widehat{q}_{i}(T, a)=-\frac{e^{\lambda T} u_{i}(T, a)}{\varepsilon}, \quad a \in(0, A) \quad 1 \leq i \leq 3 ; \\
\widehat{q}_{4}(T, a)=0, \quad a \in(0, A) .
\end{array}\right.
$$

For the proof of Lemma 2, we need an auxiliary result. This result is given in the following proposition.

Proposition 1. Let $\widehat{q}=\left(\widehat{q}_{1}, \widehat{q}_{2}, \widehat{q}_{2}\right) \in\left(L^{2}((0, T) \times(0, A))\right)^{3}$ be solution of (36) and (37). Then,

$$
\begin{aligned}
& \int_{0}^{A}\left|\widehat{q}_{1}(0, a)\right|^{2}+\left|\hat{q}_{2}(0, a)\right|^{2}+\left|\widehat{q}_{3}(0, a)\right|^{2} \mathrm{~d} a \\
& \leq K^{\prime} \int_{[0, T] \times \omega}\left|\widehat{q}_{1}\right|^{2}+\left|\hat{q}_{2}\right|^{2}+\left|\widehat{q}_{3}\right|^{2} \mathrm{~d} t \mathrm{~d} a .
\end{aligned}
$$

Proof of Proposition 1. Remark that

$$
\begin{aligned}
& \varphi^{3} e^{2 s \tilde{\alpha}} \geq \frac{e^{-2 C\left(1+(1 / T)^{2}\right)}}{T^{6}}, \quad t \in[(T / 4),(3 T / 4)], \\
& \varphi^{3} e^{2 s \alpha} \leq B_{1} e^{3 C}, \quad t \in[0, T] . \\
& \text { Using (39), (40), and (16), it follows that } \\
& \int_{\Omega \times[(T / 4),(3 T / 4)]}\left(\widehat{q}_{i}\right)^{2} \mathrm{~d} t \mathrm{~d} a \leq K_{1} \int_{[0, T] \times \omega}\left(\widehat{q}_{i}\right)^{2} \mathrm{~d} t \mathrm{~d} a .
\end{aligned}
$$

Let $\theta \in C^{2}([0, T])$ be a cut-off function such that 


$$
\left\{\begin{array}{l}
\theta(t)=1 \quad t \in[0,(T / 4)] \\
0 \leq \theta(t) \leq 1 \quad t \in[(T / 4),(3 T / 4)] \\
\theta(t)=0 \quad t>(3 T / 4)
\end{array}\right.
$$

Multiplying the equation $i$ of (36) by $\theta \widehat{q}_{i}$ and integrating by parts on $Q$, we obtain

$$
\begin{aligned}
& \int_{0}^{A}\left(\widehat{q}_{i}(0, a)\right)^{2} \mathrm{~d} a+\left(2 \lambda-C_{i}\right) \int_{Q}\left(\widehat{q}_{i}\right)^{2} \theta \mathrm{d} t \mathrm{~d} a \\
& \leq \int_{Q}-\partial_{t} \theta\left(\widehat{q}_{i}\right)^{2} \mathrm{~d} t \mathrm{~d} a .
\end{aligned}
$$

From the definition of $\theta$, it follows that

$$
\int_{0}^{A}\left(\widehat{q}_{i}(0, a)\right)^{2} \mathrm{~d} a \leq K_{2} \int_{\Omega \times[(T / 4),(3 T / 4)]}\left(\widehat{q}_{i}\right)^{2} \mathrm{~d} t \mathrm{~d} a .
$$

Next, (41) and (44) give

$$
\int_{0}^{A}\left(\widehat{q}_{i}(0, a)\right)^{2} \mathrm{~d} a \leq K^{\prime} \int_{[0, T] \times \omega}\left(\widehat{q}_{i}\right)^{2} \mathrm{~d} t \mathrm{~d} a .
$$

Finally, (45) leads to the desired result by summing with respect to $i, 1 \leq i \leq 3$.

Proof of Lemma 2.
Note that $q_{i}(t, 0)=-\int_{0}^{A}\left(\partial_{a} q_{i}\right) \mathrm{d} a$. Using Cauchy-Schwarz and Young inequalities and integrating on $[0, T]$, we obtain

$$
\int_{0}^{T}\left|q_{i}(t, 0)\right|^{2} \mathrm{~d} t \leq \frac{A}{\rho(T / 2)} \int_{0}^{T} \int_{0}^{A} \rho\left(\partial_{a} q_{i}\right)^{2} \mathrm{~d} a \mathrm{~d} t
$$

By using (16), (40), and (46), it follows that

$$
\int_{0}^{T}\left|q_{i}(t, 0)\right|^{2} \mathrm{~d} t \leq K_{s} \int_{[0, T] \times \omega} q_{i}^{2} \mathrm{~d} t \mathrm{~d} a
$$

with $K_{s}=s^{3} B_{1} e^{3 C}$. Now, as $\hat{q}_{i}(0, a)=q_{i}(0, a), \hat{q}_{i}^{2} \leq e^{2 \lambda T} q_{i}^{2}$, using (47) and Proposition 1, we get (35).

With our observability inequality, we are ready to prove the null controllability result.

Theorem 2. Assume that $(A 1)-(A 5)$ hold. Let $A>0$ and $T>0$ be given.

Then, for all $\left(u_{1}^{0}, u_{2}^{0}, u_{3}^{0}\right) \in\left(L^{2}(0, A)\right)^{3}$, there exists a control $w=\left(w_{1}, w_{2}, w_{3}\right) \in\left(L^{2}((0, T) \times \omega)\right)^{3}$ such that the associated solution of (10) and (11) verifies (4).

Proof of Theorem 2.

We consider systems (10) and (11) and the optimal control problem:

$$
\operatorname{Minimize}\left\{\frac{1}{2} \int_{Q}\left(\left|w_{1}(t, a)\right|^{2}+\left|w_{2}(t, a)\right|^{2}+\left|w_{3}(t, a)\right|^{2}\right) \mathrm{d} t \mathrm{~d} a+\frac{1}{2 \varepsilon} \int_{0}^{A}\left|u_{1}(T, a)\right|^{2}+\left|u_{2}(T, a)\right|^{2}+\left|u_{3}(T, a)\right|^{2} \mathrm{~d} a\right\}
$$

where $Q=(0, T) \times(0, A), w_{i} \in L^{2}(Q)$, and $u_{i}(1 \leq i \leq 3)$ are solutions of (10) and (11).

Let

$$
J_{\varepsilon}(w)=\frac{1}{2} \int_{Q}\left(\left|w_{1}(t, a)\right|^{2}+\left|w_{2}(t, a)\right|^{2}+\left|w_{3}(t, a)\right|^{2}\right) \mathrm{d} t \mathrm{~d} a+\frac{1}{2 \varepsilon} \int_{0}^{A}\left|u_{1}(T, a)\right|^{2}+\left|u_{2}(T, a)\right|^{2}+\left|u_{3}(T, a)\right|^{2} \mathrm{~d} a,
$$

with $w=\left(w_{1}, w_{2}, w_{3}\right)$.

The functional $J_{\varepsilon}$ is continuous, convex, and coercive. Hence, it admits a unique minimizer $w_{\varepsilon}=\left(w_{1, \varepsilon}, w_{2, \varepsilon}, w_{3, \varepsilon}\right)$, and we have after easy computations

$$
w_{i, \mathcal{\varepsilon}}(t, a)=m(a) q_{i, \mathcal{\varepsilon}}(t, a), \quad \text { a.e. }(t, a) \in \mathrm{Q}, 1 \leq i \leq 3 .
$$

Note that $\left(q_{1, \varepsilon}, q_{2, \varepsilon}, q_{3, \varepsilon}\right)$ is the solution of (12) and (13) and $u_{\varepsilon}=\left(u_{1, \varepsilon}, u_{2, \varepsilon}, u_{3, \varepsilon}\right)$ is the solution of (10) and (11) associated to $w_{\varepsilon}$.

Multiplying equation number $i$ of (12) and (13) by $u_{i, \varepsilon}$ and integrating on $Q$, it follows that

$$
\int_{Q} q_{i, \varepsilon} m(a) w_{i, \varepsilon} \mathrm{d} t \mathrm{~d} a+\frac{1}{\varepsilon} \int_{0}^{A} u_{i, \mathcal{\varepsilon}}^{2}(T, a) \mathrm{d} a+\int_{0}^{A} q_{i, \mathcal{\varepsilon}}(0, a) u_{i}^{0}(a) \mathrm{d} a+\int_{0}^{T} q_{i, \varepsilon}(t, 0) h_{i}(t) \mathrm{d} t=0 .
$$


From (51) and (50), we obtain

$$
\int_{[0, T] \times \omega} w_{i, \varepsilon}^{2} \mathrm{~d} t \mathrm{~d} a+\frac{1}{\varepsilon} \int_{0}^{A} u_{i, \mathcal{\varepsilon}}^{2}(T, a) \mathrm{d} a=-\int_{0}^{A} q_{i, \mathcal{\varepsilon}}(0, a) u_{i}^{0}(a) \mathrm{d} a-\int_{0}^{T} q_{i, \mathcal{\varepsilon}}(t, 0) h_{i}(t) \mathrm{d} t .
$$

Young inequality in (52) leads to

$$
\begin{aligned}
\int_{[0, T] \times \omega} w_{i, \varepsilon}^{2} \mathrm{~d} t \mathrm{~d} a+\frac{1}{\varepsilon} \int_{0}^{A} u_{i, \mathcal{\varepsilon}}^{2}(T, a) \mathrm{d} a \leq & \frac{1}{2 K}\left(\int_{0}^{A} q_{i, \mathcal{\varepsilon}}^{2}(0, a) \mathrm{d} a+\int_{0}^{T} q_{i, \varepsilon}^{2}(t, 0) \mathrm{d} t\right) \\
& +2 K\left(\int_{0}^{A}\left(u_{i}^{0}\right)^{2}(a) \mathrm{d} a+\int_{0}^{T} h_{i}^{2}(t) \mathrm{d} t\right) .
\end{aligned}
$$

Thus,

$$
\begin{aligned}
\int_{[0, T] \times \omega} \sum_{i=1}^{3} w_{i, \varepsilon}^{2} \mathrm{~d} t \mathrm{~d} a+\frac{1}{\varepsilon} \int_{0}^{A} \sum_{i=1}^{3} u_{i, \varepsilon}^{2}(T, a) \mathrm{d} a \leq & \frac{1}{2 K}\left(\int_{0}^{A} \sum_{i=1}^{3} q_{i, \varepsilon}^{2}(0, a) \mathrm{d} a+\int_{0}^{T} \sum_{i=1}^{3} q_{i, \varepsilon}^{2}(t, 0) \mathrm{d} t\right) \\
& +2 K\left(\int_{0}^{A} \sum_{i=1}^{3}\left(u_{i}^{0}\right)^{2}(a) \mathrm{d} a+\int_{0}^{T} \sum_{i=1}^{3} h_{i}^{2}(t) \mathrm{d} t\right) .
\end{aligned}
$$

Therefore, inequalities (35) and (54) imply

$$
\frac{1}{2} \int_{[0, T] \times \omega} \sum_{i=1}^{3} w_{i, \mathcal{\varepsilon}}^{2} \mathrm{~d} t \mathrm{~d} a+\frac{1}{\varepsilon} \int_{0}^{A} \sum_{i=1}^{3} u_{i, \mathcal{\varepsilon}}^{2}(T, a) \mathrm{d} a \leq 2 K\left(\int_{0}^{A} \sum_{i=1}^{3}\left(u_{i}^{0}\right)^{2}(a) \mathrm{d} a+\int_{0}^{T} \sum_{i=1}^{3} h_{i}^{2}(t) \mathrm{d} t\right) .
$$

From (55), we deduce

$$
\left\{\begin{array}{l}
\left\|w_{i, \varepsilon}\right\|_{L^{2}([0, T] \times \omega)}^{2} \leq 4 K\left(\int_{0}^{A} \sum_{i=1}^{3}\left(u_{i}^{0}\right)^{2}(a) \mathrm{d} a+\int_{0}^{T} \sum_{i=1}^{3} h_{i}^{2}(t) \mathrm{d} t\right), \quad 1 \leq i \leq 3, \\
\int_{0}^{A} u_{i, \varepsilon}^{2}(T, a) \mathrm{d} a \leq 2 \varepsilon K\left(\int_{0}^{A} \sum_{i=1}^{3}\left(u_{i}^{0}\right)^{2}(a) \mathrm{d} a+\int_{0}^{T} \sum_{i=1}^{3} h_{i}^{2}(t) \mathrm{d} t\right), \quad 1 \leq i \leq 3 .
\end{array}\right.
$$

Then, one can extract subsequences also denoted by $w_{i, \varepsilon}, u_{i, \varepsilon}(1 \leq i \leq 3)$ such that $w_{i, \varepsilon} \longrightarrow w_{i}$ weakly in $L^{2}((0, T) \times$ $\omega)$ and $u_{i, \varepsilon} \longrightarrow u_{i}$ weakly in $L^{2}((0, T) \times(0, A))$. Moreover, $u=\left(u_{1}, u_{2}, u_{3}\right)$ is the unique solution of (10) and (11) associated to $w=\left(w_{1}, w_{2}, w_{3}\right)$ and verifies (4).

\section{Proof of the Main Result}

Now, we consider a real number $\lambda_{0}>0$ and we set $\tilde{u}_{i}=e^{-\lambda_{0} t} u_{i}(1 \leq i \leq 4)$. Then, $\tilde{u}=\left(\tilde{u}_{1}, \widetilde{u}_{2}, \tilde{u}_{3}, \tilde{u}_{4}\right)$ is solution of the following system:

$$
\left\{\begin{array}{l}
\partial_{t} \tilde{u}_{1}+\partial_{a}\left(v_{1} \tilde{u}_{1}\right)-k \partial_{a}^{2} \widetilde{u}_{1}+\left(\lambda_{0}+\mu_{1}+\beta_{1}\right) \widetilde{u}_{1}=m(a) \widetilde{w}_{1}, \\
\partial_{t} \tilde{u}_{2}+\partial_{a}\left(v_{2} \widetilde{u}_{2}\right)-k \partial_{a}^{2} \widetilde{u}_{2}+\left(\lambda_{0}+\mu_{2}+\beta_{2}\right) \widetilde{u}_{2}=m(a) \widetilde{w}_{2}, \\
\partial_{t} \tilde{u}_{3}+\partial_{a}\left(v_{3} \tilde{u}_{3}\right)-k \partial_{a}^{2} \tilde{u}_{3}+\left(\lambda_{0}+\mu_{3}\right) \tilde{u}_{3}=m(a) \tilde{w}_{3}, \\
\partial_{t} \tilde{u}_{4}+\partial_{a}\left(v_{4} \tilde{u}_{4}\right)-k \partial_{a}^{2} \widetilde{u}_{4}+\left(\lambda_{0}+\mu_{4}\right) \tilde{u}_{4}=0,
\end{array}\right.
$$

with $(t, a) \in \Omega=(0, T) \times(0, A), \widetilde{w}_{i}=e^{-\lambda_{0} t} w_{i}$, and 


$$
\left\{\begin{array}{l}
\left(v_{1} \widetilde{u}_{1}-k \partial_{a} \widetilde{u}_{1}\right)_{a=0}=\int_{0}^{A} \beta_{3} \widetilde{u}_{3}(t, a) \mathrm{d} a, \\
\left(v_{2} \widetilde{u}_{2}-k \partial_{a} \tilde{u}_{2}\right)_{a=0}=\int_{0}^{A} \beta_{1} \widetilde{u}_{1}(t, a) \mathrm{d} a, \\
\left(v_{3} \widetilde{u}_{3}-k \partial_{a} \tilde{u}_{3}\right)_{a=0}=\int_{0}^{A} \beta_{2} \tilde{u}_{2}(t, a) \mathrm{d} a, \\
\left(v_{4} \widetilde{u}_{4}-k \partial_{a} \tilde{u}_{4}\right)_{a=0}=\int_{0}^{A}(1-\sigma) \beta_{2} \widetilde{u}_{2}(t, a) \mathrm{d} a, \\
\widetilde{u}_{i}(0, a)=\tilde{u}_{i}^{0}(a), \quad a \in \Omega_{A}=(0, A), 1 \leq i \leq 4, \\
\tilde{u}_{i}(t, A)=0, \quad t \in \Omega_{T}=(0, T), 1 \leq i \leq 4 .
\end{array}\right.
$$

Let us consider the following system:

$$
\left\{\begin{array}{l}
\partial_{t} \tilde{u}_{1}+\partial_{a}\left(v_{1} \tilde{u}_{1}\right)-k \partial_{a}^{2} \tilde{u}_{1}+\left(\lambda_{0}+\mu_{1}+\beta_{1}\right) \tilde{u}_{1}=m(a) \tilde{w}_{1}, \\
\partial_{t} \tilde{u}_{2}+\partial_{a}\left(v_{2} \tilde{u}_{2}\right)-k \partial_{a}^{2} \tilde{u}_{2}+\left(\lambda_{0}+\mu_{2}+\beta_{2}\right) \tilde{u}_{2}=m(a) \tilde{w}_{2}, \\
\partial_{t} \tilde{u}_{3}+\partial_{a}\left(v_{3} \tilde{u}_{3}\right)-k \partial_{a}^{2} \tilde{u}_{3}+\left(\lambda_{0}+\mu_{3}\right) \tilde{u}_{3}=m(a) \tilde{w}_{3}, \\
\partial_{t} \tilde{u}_{4}+\partial_{a}\left(v_{4} \tilde{u}_{4}\right)-k \partial_{a}^{2} \tilde{u}_{4}+\left(\lambda_{0}+\mu_{4}\right) \tilde{u}_{4}=0,
\end{array}\right.
$$

and

$$
\left\{\begin{array}{l}
\left(v_{1} \widetilde{u}_{1}-k \partial_{a} \widetilde{u}_{1}\right)_{a=0}=h_{1}(t) \\
\left(v_{2} \widetilde{u}_{2}-k \partial_{a} \widetilde{u}_{2}\right)_{a=0}=h_{2}(t) \\
\left(v_{3} \widetilde{u}_{3}-k \partial_{a} \widetilde{u}_{3}\right)_{a=0}=h_{3}(t) \\
\left(v_{4} \widetilde{u}_{4}-k \partial_{a} \widetilde{u}_{4}\right)_{a=0}=h_{4}(t) \\
\widetilde{u}_{i}(0, a)=\widetilde{u}_{i}^{0}(a), \quad a \in \Omega_{A}=(0, A), 1 \leq i \leq 4 \\
\tilde{u}_{i}(t, A)=0, \quad t \in \Omega_{T}=(0, T), 1 \leq i \leq 4
\end{array}\right.
$$

with $h_{1}, h_{2}, h_{3}, h_{4} \in L^{2}\left(\Omega_{T}\right)$ is defined as in (58).

Note that, from Section 3, systems (59) and (60) are null controllable. Now, we will prove the null controllability of (57) and (58).

Let $\mathscr{M}=\prod_{i=1}^{4} L^{2}\left(\Omega_{T}\right)$, and we define the following set:

$$
\begin{aligned}
\mathscr{A}_{\gamma}\left(R_{1}, R_{2}, R_{3}, R_{4}\right) & =\left\{\tilde{w}=\left(\widetilde{w}_{1}, \widetilde{w}_{2}, \widetilde{w}_{3}\right) \in \prod_{i=1}^{3} L^{2}(q), \widetilde{u} \text { is solution of }(59)-(60) \text { and }\left(\widetilde{u}_{1}, \widetilde{u}_{2}, \widetilde{u}_{3}\right) \text { verifies }(4) ; h_{i}\right. \\
& \left.=R_{i} \text { and } \widetilde{w} \text { satisfies }(56)\right\} .
\end{aligned}
$$

We introduce a multivalued function as follows:

such that

$\Lambda_{\gamma}:\left(R_{1}, R_{2}, R_{3}, R_{4}\right) \in \mathscr{M} \mapsto \Lambda_{\gamma}\left(R_{1}, R_{2}, R_{3}, R_{4}\right) \in 2^{\mathscr{M}}$,

$$
\begin{aligned}
\Lambda_{\gamma}\left(R_{1}, R_{2}, R_{3}, R_{4}\right)=\left\{\left(\int_{0}^{A} \beta_{3} \tilde{u}_{3, \widetilde{w}}(t, a) \mathrm{d} a, \int_{0}^{A} \beta_{1} \widetilde{u}_{1, \tilde{w}}(t, a) \mathrm{d} a, \int_{0}^{A} \sigma \beta_{2} \widetilde{u}_{2, \tilde{w}}(t, a) \mathrm{d} a, \int_{0}^{A}(1-\sigma) \beta_{2} \widetilde{u}_{2, \tilde{w}}(t, a) \mathrm{d} a\right):\right. \\
\left.\left(\widetilde{u}_{1, \tilde{w}}, \widetilde{u}_{2, \widetilde{w}}, \widetilde{u}_{3, \widetilde{w}}\right) \text { is solution of }(59)-(60) \text { and } \widetilde{w} \in \mathscr{A}_{\gamma}\left(R_{1}, R_{2}, R_{3}, R_{4}\right)\right\} .
\end{aligned}
$$

The goal is to prove that the multivalued function $\Lambda_{\gamma}$ admits a fixed point. To this end, we will use the generalization of Leray-Schauder fixed point theorem. Consider

$$
\begin{aligned}
\mathscr{N}_{\gamma} & =\left\{\left(R_{1}, R_{2}, R_{3}, R_{4}\right) \in \mathscr{M}: \exists \rho \in(0,1),\left(R_{1}, R_{2}, R_{3}, R_{4}\right)\right. \\
& \left.\in \rho \Lambda_{\gamma}\left(R_{1}, R_{2}, R_{3}, R_{4}\right)\right\} .
\end{aligned}
$$

The fixed point existence of the multivalued function $\Lambda_{\gamma}$ is an immediate consequence of the following proposition.

Proposition 2. (i) $\mathscr{N}_{\gamma}$ is bounded on $\mathscr{M}$ (ii) For all $\left(R_{1}, R_{2}, R_{3}, R_{4}\right) \in \mathscr{M}, \Lambda_{\gamma}\left(R_{1}, R_{2}, R_{3}, R_{4}\right)$ is a nonempty closed and convex subset of $\mathscr{M}$

(iii) $\Lambda_{\gamma}: \mathscr{M} \longrightarrow 2^{\mathscr{M}}$ is compact

(iv) The multivalued mapping $\Lambda_{\gamma}$ is upper semicontinuous on $\mathscr{M}$

Proof of Proposition 2.

Let us set $\left(\tilde{u}_{1, \tilde{w}}, \tilde{u}_{2, \tilde{w}}, \tilde{u}_{3, \tilde{w}}, \tilde{u}_{4, \tilde{w}}\right)=\left(\tilde{u}_{1}, \tilde{u}_{2}, \tilde{u}_{3}, \tilde{u}_{4}\right)$. (i) Let $\left(R_{1}, R_{2}, R_{3}, R_{4}\right) \in \mathcal{N}_{\gamma}$. There is $\rho \in(0,1)$ such that $(1 / \rho)\left(R_{1}, R_{2}, R_{3}, R_{4}\right) \in \Lambda_{\gamma}\left(R_{1}, R_{2}, R_{3}, R_{4}\right)$. Hence, there is $\left(\widetilde{u}_{1}, \widetilde{u}_{2}, \widetilde{u}_{3}\right) \in \prod_{i=1}^{3} L^{2}(\Omega)$ associated to $\widetilde{w} \in \prod_{i=1}^{3} L^{2}(q)$ such that 


$$
\begin{aligned}
& R_{1}=\int_{0}^{A} \beta_{3} \tilde{u}_{3} \mathrm{~d} a, \\
& R_{2}=\int_{0}^{A} \beta_{1} \widetilde{u}_{1} \mathrm{~d} a, \\
& R_{3}=\int_{0}^{A} \beta_{2} \tilde{u}_{2} \mathrm{~d} a, \\
& R_{4}=\int_{0}^{A} \beta_{2} \tilde{u}_{2} \mathrm{~d} a,
\end{aligned}
$$

with $\left(\widetilde{u}_{1}, \widetilde{u}_{2}, \widetilde{u}_{3}\right)$ solution of (57) and (58) associated to $\widetilde{w}$ satisfying (56). Then, we have

$$
\sum_{i=1}^{4}\left\|R_{i}\right\|_{L^{2}\left(\Omega_{T}\right)}^{2} \leq \sum_{i=1}^{4} \xi_{i}\left\|\tilde{u}_{i}\right\|_{L^{2}(\Omega)}^{2} \xi_{i}=A\left\|\beta_{i}\right\|_{L^{\infty}([0, A])} \text { and } \xi_{4}=0 .
$$

Multiplying the equation number $i$ of (57) and (58) by $\widetilde{u}_{i}$ and integrating by parts on $\Omega$, we obtain after summing with respect to $i(1 \leq i \leq 4)$ :

$$
\begin{aligned}
\sum_{i=1}^{4}\left(2 \lambda_{0}-C_{i}-1\right)\left\|\widetilde{u}_{i}\right\|_{L^{2}(\Omega)}^{2} \leq & \sum_{i=1}^{4}\left\|\widetilde{u}_{i}^{0}\right\|_{L^{2}([0, A])}^{2}+\sum_{i=1}^{4}\left\|R_{i}\right\|_{L^{2}\left(\Omega_{T}\right)}^{2} \\
& +\sum_{i=1}^{4}\left\|\widetilde{w}_{i}\right\|_{L^{2}(q)}^{2}, \quad\left(w_{4} \equiv 0\right) .
\end{aligned}
$$

In virtue of (66), (67), and (56), it follows that

$$
\begin{aligned}
\sum_{i=1}^{4}\left(2 \lambda_{0}-C_{i}-1\right)\left\|\tilde{u}_{i}\right\|_{L^{2}(\Omega)}^{2} \leq & (4 K+1) \sum_{i=1}^{4}\left\|\tilde{u}_{i}^{0}\right\|_{L^{2}([0, A])}^{2} \\
& +(4 K+1) \sum_{i=1}^{4} \xi_{i}\left\|\tilde{u}_{i}\right\|_{L^{2}(\Omega)}^{2} .
\end{aligned}
$$

Inequality (68) leads to

$$
\sum_{i=1}^{4}\left(2 \lambda_{0}-C_{i}-1-(4 K+1) \xi_{i}\right)\left\|\tilde{u}_{i}\right\|_{L^{2}(\Omega)}^{2} \leq(4 K+1) \sum_{i=1}^{4}\left\|\tilde{u}_{i}^{0}\right\|_{L^{2}([0, A])}^{2} .
$$

Considering again (66) and (69), we get the desired result. ( $i i)\left(R_{1}, R_{2}, R_{3}, R_{4}\right) \in \mathscr{M}, \Lambda_{\gamma}\left(R_{1}, R_{2}, R_{3}, R_{4}\right)$ is nonempty. In fact, systems (57) and (58) are equivalent to systems (10) and (11) which admits a solution. Also, $\widetilde{w}$ satisfies (56).

Since the function $\left(R_{1}, R_{2}, R_{3}, R_{4}\right) \mapsto\left(\widetilde{u}_{1}, \widetilde{u}_{2}, \widetilde{u}_{3}, \widetilde{u}_{4}\right)$ is affine, it follows that $\Lambda_{\gamma}\left(R_{1}, R_{2}, R_{3}, R_{4}\right)$ is convex.

Let $\left(\eta_{n}^{1}, \eta_{n}^{2}, \eta_{n}^{3}, \eta_{n}^{4}\right) \in \Lambda_{\gamma}\left(R_{1}, R_{2}, R_{3}, R_{4}\right)$ such that $\left(\eta_{n}^{1}, \eta_{n}^{2}, \eta_{n}^{3}, \eta_{n}^{4}\right) \longrightarrow\left(\eta^{1}, \eta^{2}, \eta^{3}, \eta^{4}\right)$ in $\mathscr{M}$. Thus, for all $n$, there exists $\widetilde{w}_{n}$ which satisfies (56), such that

$$
\begin{aligned}
\eta_{n}^{1} & =\int_{0}^{A} \beta_{3} \widetilde{u}_{3, n} \mathrm{~d} a, \\
\eta_{n}^{2} & =\int_{0}^{A} \beta_{1} \widetilde{u}_{1, n} \mathrm{~d} a, \\
\eta_{n}^{3} & =\int_{0}^{A} \sigma \beta_{2} \widetilde{u}_{2, n} \mathrm{~d} a, \\
\eta_{n}^{4} & =\int_{0}^{A}(1-\sigma) \beta_{2} \widetilde{u}_{2, n} \mathrm{~d} a .
\end{aligned}
$$

Here, $\left(\widetilde{u}_{1, n}, \widetilde{u}_{2, n}, \widetilde{u}_{3, n}\right)$ is solution of (59) and (60), with $\left(R_{1}, R_{2}, R_{3}, R_{4}\right)$ instead of $\left(h_{1}, h_{2}, h_{3}, h_{4}\right)$ and $\left(\tilde{u}_{1, n}, \tilde{u}_{2, n}, \tilde{u}_{3, n}\right)$ which verify (69). From (66), (69), and (56), one can extract sequences still denoted by $\left(\left(\widetilde{u}_{1, n}, \widetilde{u}_{2, n}, \widetilde{u}_{3, n}\right)\right)_{n}$ and $\left(\widetilde{w}_{n}\right)_{n}$ that converge weakly towards $\left(\widetilde{u}_{1}, \widetilde{u}_{2}, \widetilde{u}_{3}\right)$ and $\widetilde{w}$ in $\left(L^{2}(\Omega)\right)^{3}$ and in $\left(L^{2}(q)\right)^{3}$, respectively.

So,

$$
\begin{aligned}
& \eta^{1}=\int_{0}^{A} \beta_{3} \tilde{u}_{3} \mathrm{~d} a, \\
& \eta^{2}=\int_{0}^{A} \beta_{1} \tilde{u}_{1} \mathrm{~d} a, \\
& \eta^{3}=\int_{0}^{A} \sigma \beta_{2} \tilde{u}_{2} \mathrm{~d} a, \\
& \eta^{4}=\int_{0}^{A}(1-\sigma) \beta_{2} \widetilde{u}_{2} \mathrm{~d} a .
\end{aligned}
$$

Moreover, $\left(\widetilde{u}_{1}, \widetilde{u}_{2}, \widetilde{u}_{3}\right)$ is solution of (59) and (60), with $\left(R_{1}, R_{2}, R_{3}, R_{4}\right)=\left(h_{1}, h_{2}, h_{3}, h_{4}\right)$. Also, $\tilde{w}$ verifies (56) and $\left(\tilde{u}_{1}, \tilde{u}_{2}, \tilde{u}_{3}\right)$ verifies (4). Then, $\left(\eta^{1}, \eta^{2}, \eta^{3}, \eta^{4}\right) \in \Lambda_{\gamma}\left(R_{1}, R_{2}, R_{3}\right.$, $\left.R_{4}\right)$. (iii) Let $\left(R_{1}, R_{2}, R_{3}, R_{4}\right) \in K$ a bounded subset of $\mathscr{M}$. Then, there exist $r_{i}>0(1 \leq i \leq 4)$ such that

$$
\left\|R_{i}\right\|_{L^{2}\left(Q_{T}\right)} \leq r_{i}, \quad 1 \leq i \leq 4 .
$$

Consider $\left(\left(\eta_{n}^{1}, \eta_{n}^{2}, \eta_{n}^{3}, \eta_{n}^{4}\right)\right)_{n} \in \Lambda_{\gamma}\left(R_{1}, R_{2}, R_{3}, R_{4}\right)$; for all $n \in \mathbb{N}$, there is $\left(\widetilde{u}_{1, n}, \widetilde{u}_{2, n}, \widetilde{u}_{3, n}\right) \in\left(L^{2}(\Omega)\right)^{3}$ and a control function $\widetilde{w}_{n} \in\left(L^{2}(q)\right)^{3}$ such that

$$
\begin{aligned}
& \eta_{n}^{1}=\int_{0}^{A} \beta_{3} \widetilde{u}_{3, n} \mathrm{~d} a, \\
& \eta_{n}^{2}=\int_{0}^{A} \beta_{1} \widetilde{u}_{1, n} \mathrm{~d} a, \\
& \eta_{n}^{3}=\int_{0}^{A} \sigma \beta_{2} \widetilde{u}_{2, n} \mathrm{~d} a, \\
& \eta_{n}^{4}=\int_{0}^{A}(1-\sigma) \beta_{2} \tilde{u}_{2, n} \mathrm{~d} a,
\end{aligned}
$$

where $\left(\widetilde{u}_{1, n}, \widetilde{u}_{2, n}, \widetilde{u}_{3, n}\right)$ is solution of (59) and (60) with $\left(R_{1, n}, R_{2, n}, R_{3, n}, R_{4, n}\right)$ instead of $\left(h_{1}, h_{2}, h_{3}, h_{4}\right)$ and $\widetilde{w}_{n}$ verifies (56). Using (56), it follows that

$$
\int \sum_{i=1}^{3} \widetilde{w}_{i, n}^{2}(t, a) \mathrm{d} t \mathrm{~d} a \leq 4 K\left(\int_{0}^{A} \sum_{i=1}^{4}\left(\widetilde{u}_{i}^{0}\right)^{2} \mathrm{~d} a+\sum_{i=1}^{4} r_{i}^{2}\right) .
$$


Therefore, $\left(\widetilde{w}_{n}\right)$ is bounded, and we can extract a sequence still denoted by $\left(\widetilde{w}_{n}\right)$ that weakly converges towards $\widetilde{w}$ in $L^{2}(q)$.

Now, multiplying the equation number $i$ of (59) and (60) by $\tilde{u}_{i, n}$, integrating by parts on $Q$ and using (72), we get after summing

$$
\begin{aligned}
& \int_{Q} \sum_{i=1}^{4} k\left(\left(\widetilde{u}_{i, n}\right)_{a}\right)^{2}(t, a)+\widetilde{u}_{i, n}^{2}(t, a) \mathrm{d} t \mathrm{~d} a \\
& \leq(4 K+1)\left(\int_{0}^{A} \sum_{i=1}^{4}\left(\widetilde{u}_{i}^{0}\right)^{2} \mathrm{~d} a+\sum_{i=1}^{4} r_{i}^{2}\right) .
\end{aligned}
$$

Consequently, the sequence $\left(\widetilde{u}_{1, n}, \widetilde{u}_{2, n}, \widetilde{u}_{3, n}\right)$ is bounded in $\left(L^{2}(Q)\right)^{3}$. One can extract a sequence denoted $\left(\left(\widetilde{u}_{1, n_{k}}, \widetilde{u}_{2, n_{k}}, \widetilde{u}_{3, n_{k}}\right)\right)$ that converges weakly towards $\left(\widetilde{u}_{1}, \widetilde{u}_{2}, \widetilde{u}_{3}\right)$ in $\left(L^{2}(Q)\right)^{3}$.

As

$$
\begin{aligned}
\eta_{n_{k}}^{1} & =\int_{0}^{A} \beta_{3} \widetilde{u}_{3, n_{k}} \mathrm{~d} a, \\
\eta_{n_{k}}^{2} & =\int_{0}^{A} \beta_{1} \widetilde{u}_{1, n_{k}} \mathrm{~d} a, \\
\eta_{n_{k}}^{3} & =\int_{0}^{A} \sigma \beta_{2} \widetilde{u}_{2, n_{k}} \mathrm{~d} a, \\
\eta_{n_{k}}^{4} & =\int_{0}^{A}(1-\sigma) \beta_{2} \widetilde{u}_{2, n_{k}} \mathrm{~d} a,
\end{aligned}
$$

using (75), we obtain

$$
\sum_{i=1}^{4}\left\|\eta_{n_{k}}^{i}\right\|_{L^{2}\left(Q_{T}\right)} \leq K^{\prime}\left(\int_{0}^{A} \sum_{i=1}^{4}\left(\widetilde{u}_{i}^{0}\right)^{2} \mathrm{~d} a+\sum_{i=1}^{4} r_{i}^{2}\right) .
$$

From (77), one can extract a sequence which also denoted $\left(\left(\eta_{n_{k}}^{1}, \eta_{n_{k}}^{2}, \eta_{n_{k}}^{3}, \eta_{n_{k}}^{4}\right)\right)$ that converges weakly to $\left(\eta^{1}, \eta^{2}, \eta^{3}, \eta^{4}\right)$ on $L^{2}\left(Q_{T}\right)$.

So,

$$
\int_{Q_{T}} \phi \eta_{n_{j}}^{i} \mathrm{~d} t \longrightarrow \int_{Q_{T}} \phi \eta^{i} \mathrm{~d} t, \quad \forall \phi \in L^{2}\left(Q_{T}\right), 1 \leq i \leq 4 .
$$

Moreover, the sequence $\left(\left(\widetilde{u}_{1, n_{k}}, \widetilde{u}_{2, n_{k}}, \widetilde{u}_{3, n_{k}}\right)\right)$ converges weakly towards $\left(\widetilde{u}_{1}, \widetilde{u}_{2}, \widetilde{u}_{3}\right)$ on $\left(L^{2}(Q)\right)^{3}$. Thus, the sequence $\left(\left(\widetilde{u}_{1, n_{k}}, \widetilde{u}_{2, n_{k}}, \widetilde{u}_{3, n_{k}}\right)\right)$ associated to $\left(\left(\eta_{n_{k}}^{1}, \eta_{n_{k}}^{2}, \eta_{n_{k}}^{3}, \eta_{n_{k}}^{4}\right)\right)$ converges weakly towards $\left(\widetilde{u}_{1}, \widetilde{u}_{2}, \widetilde{u}_{3}\right)$. Since $\phi \beta_{k} \in L^{2}\left(Q_{T}\right)$, for all $\phi \in L^{2}\left(Q_{T}\right)$ and for all $k \in\{1,2,3\}$, it follows that

$$
\left\{\begin{array}{l}
\int_{Q_{T}} \phi \eta_{n_{k}}^{1} \mathrm{~d} t \longrightarrow \int_{Q_{T}} \phi\left(\int_{0}^{A} \beta_{3} \widetilde{u}_{3} d a\right) \mathrm{d} t \\
\int_{Q_{T}} \phi \eta_{n_{k}}^{2} \mathrm{~d} t \longrightarrow \int_{Q_{T}} \phi\left(\int_{0}^{A} \beta_{1} \widetilde{u}_{1} d a\right) \mathrm{d} t, \\
\int_{Q_{T}} \phi \eta_{n_{k}}^{3} \mathrm{~d} t \longrightarrow \int_{Q_{T}} \phi\left(\int_{0}^{A} \sigma \beta_{2} \widetilde{u}_{2} d a\right) \mathrm{d} t, \\
\int_{Q_{T}} \phi \eta_{n_{k}}^{4} \mathrm{~d} t \longrightarrow \int_{Q_{T}} \phi\left(\int_{0}^{A}(1-\sigma) \beta_{2} \widetilde{u}_{2} d a\right) \mathrm{d} t .
\end{array}\right.
$$

Equations (78) and (79) imply

$$
\begin{cases}\int_{Q_{T}} \phi\left(\eta^{1}-\int_{0}^{A} \beta_{3} \widetilde{u}_{3} \mathrm{~d} a\right) \mathrm{d} t=0, & \forall \phi \in L^{2}\left(Q_{T}\right), \\ \int_{Q_{T}} \phi\left(\eta^{2}-\int_{0}^{A} \beta_{1} \widetilde{u}_{1} \mathrm{~d} a\right) \mathrm{d} t, & \forall \phi \in L^{2}\left(Q_{T}\right), \\ \int_{Q_{T}} \phi\left(\eta^{3}-\int_{0}^{A} \sigma \beta_{2} \widetilde{u}_{2} \mathrm{~d} a\right) \mathrm{d} t=0, & \forall \phi \in L^{2}\left(Q_{T}\right), \\ \int_{Q_{T}} \phi\left(\eta^{4}-\int_{0}^{A}(1-\sigma) \beta_{2} \widetilde{u}_{2} \mathrm{~d} a\right) \mathrm{d} t=0, & \forall \phi \in L^{2}\left(Q_{T}\right) .\end{cases}
$$

Consequently,

$$
\left\{\begin{array}{l}
\eta^{1}=\int_{0}^{A} \beta_{3} \widetilde{u}_{3} \mathrm{~d} a, \quad \text { a.e. } t \in Q_{T}, \\
\eta^{2}=\int_{0}^{A} \beta_{1} \widetilde{u}_{1} \mathrm{~d} a, \quad \text { a.e. } t \in Q_{T}, \\
\eta^{3}=\int_{0}^{A} \sigma \beta_{2} \widetilde{u}_{2} \mathrm{~d} a, \quad \text { a.e. } t \in Q_{T}, \\
\eta^{4}=\int_{0}^{A}(1-\sigma) \beta_{2} \widetilde{u}_{2} \text { da, } \quad \text { a.e. } t \in Q_{T} .
\end{array}\right.
$$

Then, $\left(\widetilde{u}_{1}, \widetilde{u}_{2}, \widetilde{u}_{3}, \widetilde{u}_{4}\right)$ is solution of (59) and (60) with $\left(R_{1}, R_{2}, R_{3}, R_{4}\right)$ instead of $\left(h_{1}, h_{2}, h_{3}, h_{4}\right) ;\left(\widetilde{u}_{1}, \widetilde{u}_{2}, \widetilde{u}_{3}\right)$ and $\widetilde{w}$ satisfy, respectively, (4) and (56). (iv) Let $K$ be a closed subset of $\mathscr{M}$. We will prove that $\Lambda_{\gamma}^{-1}(K)$ is a closed subset of $\mathscr{M}$. Remark that $\Lambda_{\gamma}^{-1}(K)=\left\{\left(R_{1}, R_{2}, R_{3}, R_{4}\right) \in \mathscr{M} ; \Lambda_{\gamma}\left(R_{1}, R_{2}\right.\right.$, $\left.\left.R_{3}, R_{4}\right) \cap K \neq \varnothing\right\}$.

Suppose that $\left(R_{1, n}, R_{2, n}, R_{3, n}, R_{4, n}\right) \longrightarrow\left(R_{1}, R_{2}, R_{3}, R_{4}\right)$ (strongly) in $\mathscr{M}$. Then, the sequence $\left(R_{1, n}, R_{2, n}, R_{3, n}, R_{4, n}\right)$ is bounded. There also exists a sequence $\left(\left(\eta_{n}^{1}, \eta_{n}^{2}, \eta_{n}^{3}, \eta_{n}^{4}\right)\right)_{n} \in K$, such that, for all $n \geq 1,\left(\eta_{n}^{1}, \eta_{n}^{2}, \eta_{n}^{3}, \eta_{n}^{4}\right) \in \Lambda_{\gamma}\left(R_{1, n}, R_{2, n}\right.$, 

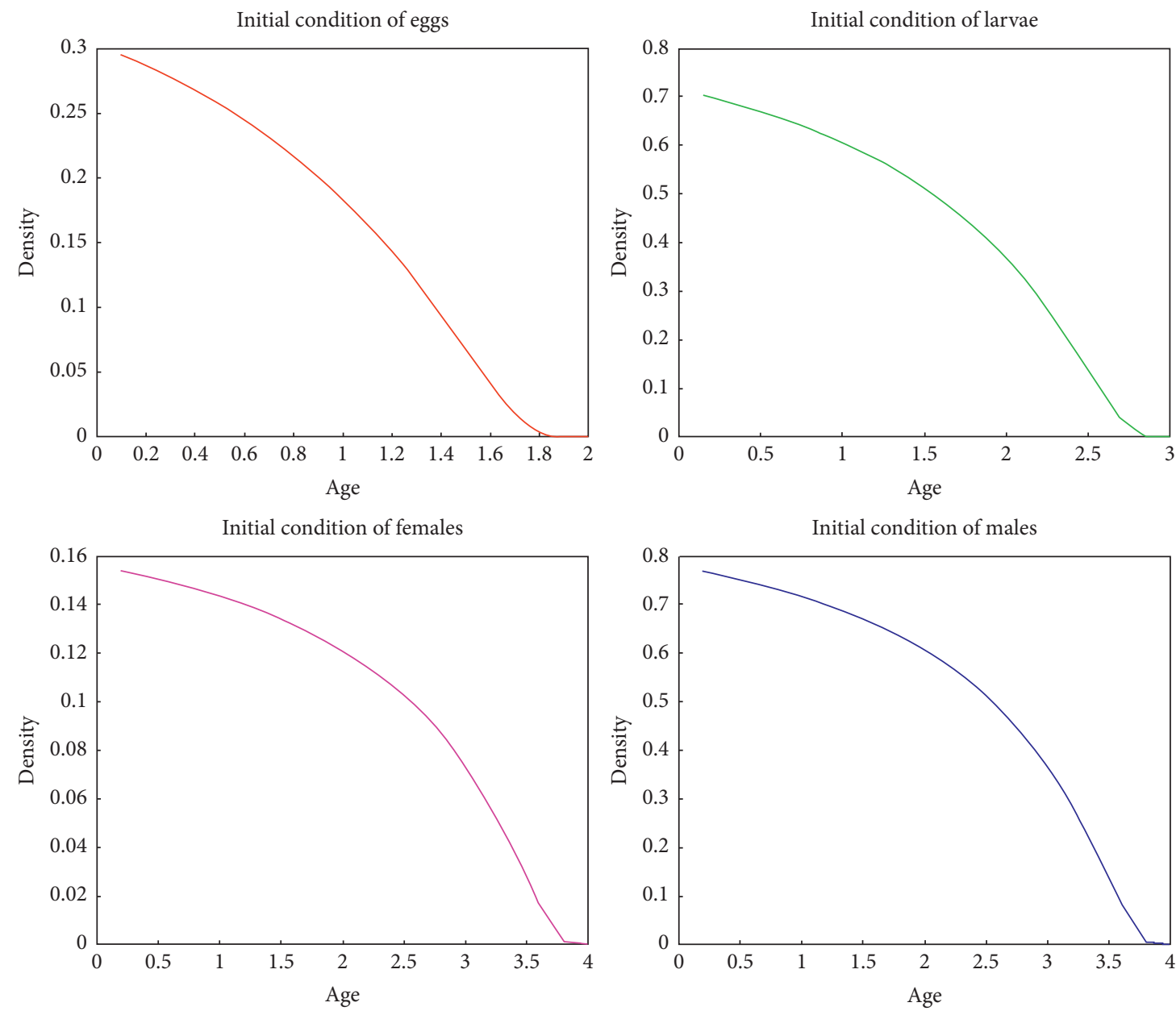

FIgURE 1: Initial conditions.

$\left.R_{3, n}, R_{4, n}\right)$. So, there exist $\left(\tilde{u}_{1, n}, \tilde{u}_{2, n}, \tilde{u}_{3, n}\right) \in\left(L^{2}(\Omega)\right)^{3}$ and a control function $\widetilde{w}_{n} \in\left(L^{2}(q)\right)^{3}$ such that

$$
\begin{aligned}
\eta_{n}^{1} & =\int_{0}^{A} \beta_{3} \tilde{u}_{3, n} \mathrm{~d} a, \\
\eta_{n}^{2} & =\int_{0}^{A} \beta_{1} \tilde{u}_{1, n} \mathrm{~d} a, \\
\eta_{n}^{3} & =\int_{0}^{A} \sigma \beta_{2} \widetilde{u}_{2, n} \mathrm{~d} a, \\
\eta_{n}^{4} & =\int_{0}^{A}(1-\sigma) \beta_{2} \tilde{u}_{2, n} \mathrm{~d} a,
\end{aligned}
$$

with $\widetilde{w}_{n},\left(\widetilde{u}_{1, n}, \widetilde{u}_{2, n}, \widetilde{u}_{3, n}\right)$ verifying (56) and $\left(\widetilde{u}_{1, n}, \widetilde{u}_{2, n}, \widetilde{u}_{3, n}\right)$ is solution of (59) and (60) with $\left(R_{1, n}, R_{2, n}, R_{3, n}, R_{4, n}\right)$ instead of $\left(h_{1}, h_{2}, h_{3}, h_{4}\right)$, and $\left(\widetilde{u}_{1, n}, \widetilde{u}_{2, n}, \widetilde{u}_{3, n}\right)$ verifies (4). From (66), (69), and (56), it follows that $\left(\widetilde{w}_{n}\right)$ and $\left(\widetilde{u}_{1, n}, \widetilde{u}_{2, n}, \widetilde{u}_{3, n}\right)$ are bounded, respectively, in $\left(L^{2}(q)\right)^{3}$ and $\left(L^{2}(\Omega)\right)^{3}$. Thus, there are sequences also denoted by $\left(\widetilde{w}_{n}\right)_{n}$ and $\left(\left(\widetilde{u}_{1, n}, \widetilde{u}_{2, n}, \widetilde{u}_{3, n}\right)\right)_{n}$ that converge weakly towards $\widetilde{w}$ and $\left(\widetilde{u}_{1}, \widetilde{u}_{2}, \widetilde{u}_{3}\right)$, respectively, in $\left(L^{2}(q)\right)^{3}$ and $\left(L^{2}(\Omega)\right)^{3}$. Moreover, $\widetilde{w}$ verifies $(56)$ and

$$
\begin{aligned}
\eta^{1} & =\int_{0}^{A} \beta_{3} \tilde{u}_{3} d a, \\
\eta^{2} & =\int_{0}^{A} \beta_{1} \tilde{u}_{1} d a, \\
\eta^{3} & =\int_{0}^{A} \sigma \beta_{2} \tilde{u}_{2} d a, \\
\eta^{4} & =\int_{0}^{A}(1-\sigma) \beta_{2} \tilde{u}_{2} d a .
\end{aligned}
$$

Here, $\left(\widetilde{u}_{1}, \widetilde{u}_{2}, \widetilde{u}_{3}\right)$ is solution of (59) and (60) with $\left(R_{1}, R_{2}, R_{3}, R_{4}\right)$ instead of $\left(h_{1}, h_{2}, h_{3}, h_{4}\right)$, and $\left(\tilde{u}_{1}, \tilde{u}_{2}, \tilde{u}_{3}\right)$ verifies (4). Therefore,

$$
\left(\eta^{1}, \eta^{2}, \eta^{3}, \eta^{4}\right) \in \Lambda_{\gamma}\left(R_{1}, R_{2}, R_{3}, R_{4}\right) .
$$

In addition, from (77) and Lions-Aubin lemma, one can extract a sequence $\left(\left(\eta_{n}^{1}, \eta_{n}^{2}, \eta_{n}^{3}, \eta_{n}^{4}\right)\right)_{n}$ that converges strongly to $\left(\eta^{1}, \eta^{2}, \eta^{3}, \eta^{4}\right)$ in $\mathscr{M}$. Since $K$ is closed, then $\left(\eta^{1}, \eta^{2}, \eta^{3}, \eta^{4}\right) \in K$.

Finally, from (84), we conclude that $\left(R_{1}, R_{2}, R_{3}, R_{4}\right) \in$ $\Lambda_{\gamma}^{-1}(K)$. 

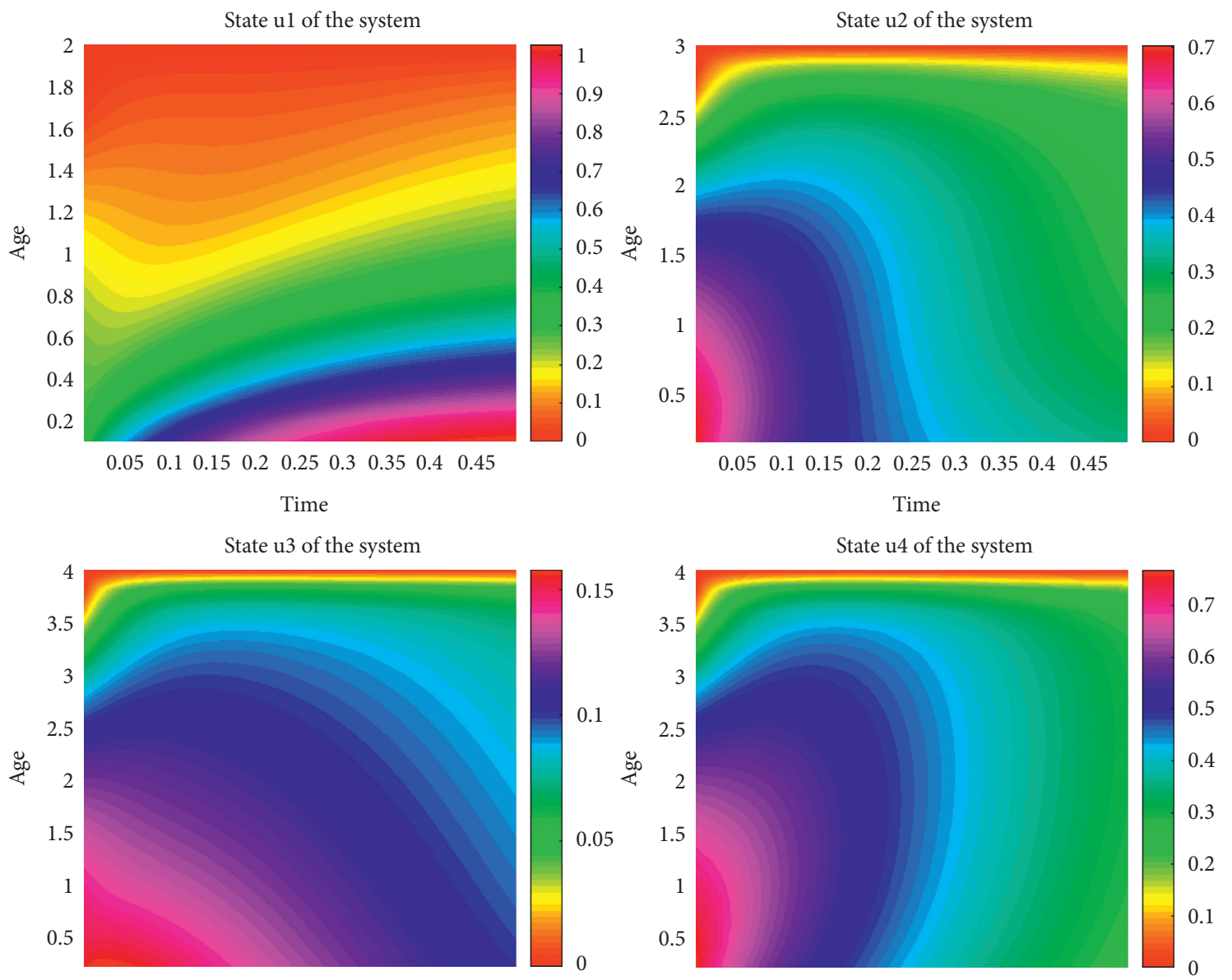

$\begin{array}{lllllllll}0.05 & 0.1 & 0.15 & 0.2 & 0.25 & 0.3 & 0.35 & 0.4 & 0.45\end{array}$

Time

$\begin{array}{lllllllll}0.05 & 0.1 & 0.15 & 0.2 & 0.25 & 0.3 & 0.35 & 0.4 & 0.45\end{array}$

Time

Figure 2: Uncontrolled solution at final time $T=0.5$ and $A=4$.

Proof. of Theorem 1.

This result is a consequence of Proposition 2. In fact, from Proposition 2, there is $\left(R_{1}, R_{2}, R_{3}, R_{4}\right) \in \mathscr{M}$ such that $\left(R_{1}\right.$, $\left.R_{2}, R_{3}, R_{4}\right) \in \Lambda_{\gamma}\left(R_{1}, R_{2}, R_{3}, R_{4}\right)$, see Theorem 1.3 in [22].

\section{Numerical Simulations}

In this section, we display the numerical simulations of problems (1) and (2). The first part is to reduce the PDE to a finite dimensional system of the form: $\dot{X}=A X+B Y$, where $A$ and $B$ are matrices. We construct the control problem, which consists in minimizing the functional and we choose the classical Hum functional and the control matrix $B=\chi_{\Theta}$, where $\Theta=(0, A) \times(0, T)$.

Example 1. For the simulation, we take $k=0.25$, $A_{1}=2, A_{2}=3, A_{3}=A_{4}=4$, and $\Delta a=1 / 20$. Moreover, the transition functions $\beta_{1}, \beta_{2}$, and $\beta_{3}$ are given by $\beta_{1}(a)= \begin{cases}0, & \text { if } a=0, \\ \frac{7(11 a-4)^{4} e^{-0.52(10 a-4)}}{\Gamma(5)}, & \text { if } a \neq 0,\end{cases}$

$$
\beta_{2}(a)= \begin{cases}0, & \text { if } a=0, \\ \frac{7(50 a-4)^{4} e^{-0.52(10 a-4)}}{\Gamma(5)}, & \text { if } a \neq 0,\end{cases}
$$

$$
\beta_{3}(a)= \begin{cases}0, & \text { if } a=0, \\ \frac{7\left(12.10^{6} a-4\right)^{3} e^{-0.52(10 a-4)}}{\Gamma(5)}, & \text { if } a \neq 0,\end{cases}
$$



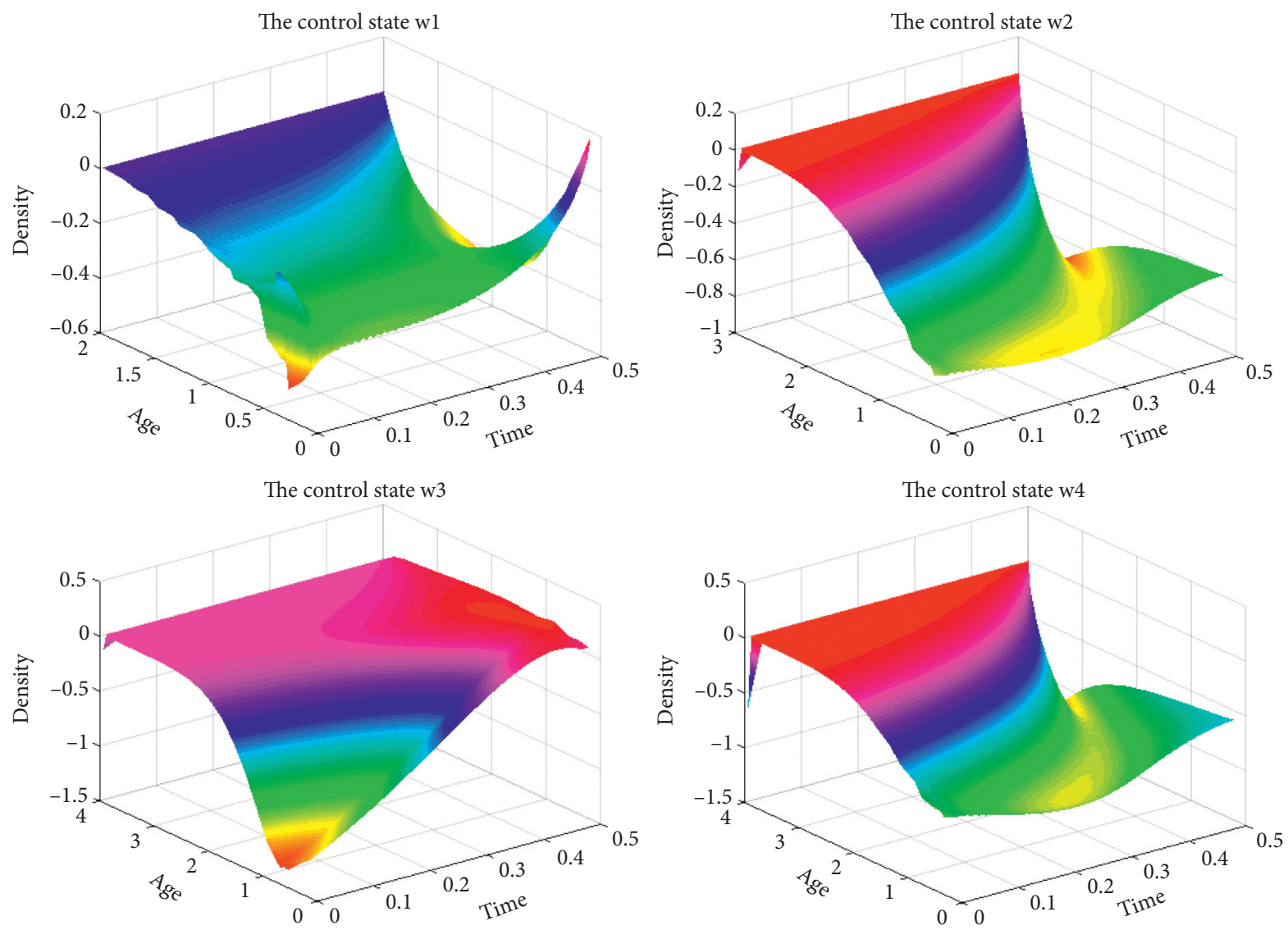

Figure 3: Control state at final time $T=0.5$ and $A=4$.

and the initial population distributions $u_{i}^{0}(a)=k_{i} e^{-\left(1 / A_{i}-a\right)},\left(1 \leq i \leq 4\right.$, with $\mathrm{k}_{1}=0.5, k_{2}=k_{4}=$ 1 and $k_{3}=0.2$ ) will be used in the computation. The growth rates are given by

$$
\begin{aligned}
& v_{1}(a)=\left\{\begin{array}{lll}
0.2, & \text { if } & a=0, \\
a, & \text { if } & a \neq 0,
\end{array}\right. \\
& v_{2}(a)=\left\{\begin{array}{lll}
0.3, & \text { if } & a=0, \\
a, & \text { if } & a \neq 0,
\end{array}\right. \\
& v_{3}(a)=\left\{\begin{array}{lll}
0.4, & \text { if } & a=0, \\
a, & \text { if } & a \neq 0,
\end{array}\right. \\
& v_{4}(a)=\left\{\begin{array}{lll}
0.4, & \text { if } & a=0, \\
a, & \text { if } & a \neq 0 .
\end{array}\right.
\end{aligned}
$$

The following numerical results were obtained.

We notice that we have the positivity of the initial conditions, see Figure 1. Moreover, the colors green, blue, and yellow show that the uncontrolled solutions are not null at the final time $T=0.5$ and the color red shows that the uncontrolled solutions are null at the final age $A=4$, see Figure 2.

We use ODE 45 for the simulations of the uncontrolled system.

Example 2. In this example, we are going back to the data from Example 1.

The control states and the controlled states results are obtained for $T=0.5$ and $A=4$.

We notice that numerically the control covers all the domains. However, we could not take a positivity constraint in our simulations, see Figure 3.

Moreover, the colors red and yellow show that the controlled states are zero at the final time $T=0.5$, see Figure 4. This confirms the result of null controllability obtained theoretically.

The CaSadi toolbox is used to simulate the control (by the minimization of functional: Hum Method).

Below, we have the numerical simulations results. 

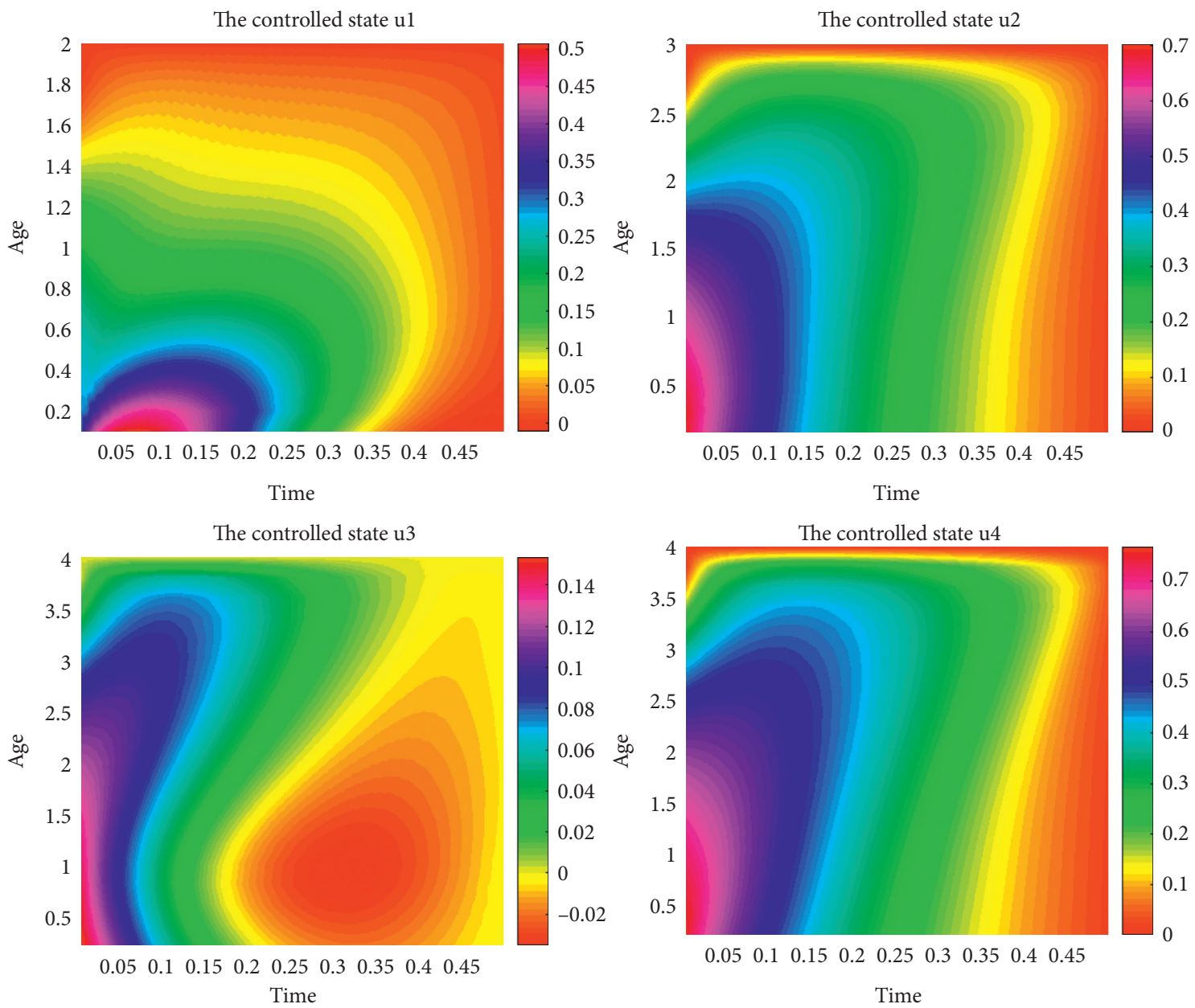

Figure 4: Controlled solution at final time $T=0.5$ and $A=4$.

\section{Conclusion}

In this paper, we have proved the null controllability of a four-stage and age-structured population dynamics model. We consider three controls acting on eggs, larvae, and females. We end with numerical illustrations to confirm the theoretical results. In order to consolidate the result obtained in this article, we are going to think about studying the controllability with less controls (two or one) and also to establish algorithms allowing to calculate the control from its characterization.

\section{Data Availability}

No data were used to support this study.

\section{Conflicts of Interest}

The authors declare that they have no conflicts of interest.

\section{References}

[1] A. S. Balachowsky, Entomologie appliquée à l'agriculture, Masson, Paris, France, 1962-1963.

[2] P. A. Ouedraogo, J. P. Monge, and J. Huignard, "Importance of temperature and seed water content on the induction of imaginal polymorphism in Callosobruchus maculatus," Entomologia Experimentalis et Applicata, vol. 59, no. 1, pp. 59-66, 1991.

[3] S. Aniţa, Analysis and Control of Age-dependent Population Dynamics, Kluwer Academic Publishers, Amsterdam, Netherlands, 2000.

[4] Z. Luo, Z.-R. He, and W.-T. Li, "Optimal birth control for an age-dependent n-dimensional food chain model," Journal of Mathematical Analysis and Applications, vol. 287, no. 2, pp. 557-576, 2003.

[5] D. Picart, B. E. Ainseba, and F. Milner, "Optimal control problem on insect pest populations," Applied Mathematics Letters, vol. 24, no. 7, pp. 1160-1164, 2011.

[6] N. H. Sweilam, S. M. AL-Mekhlafi, Z. N. Mohammed, and D. Baleanu, "Optimal control for variable order fractional HIV/AIDS and malaria mathematical models with multi-time delay," Alexandria Engineering Journal, vol. 59, no. 5, pp. 3149-3162, 2020.

[7] N. H. Sweilam, S. M. AL-Mekhlafi, A. O. Albalaw, and D. Baleanu, "On the optimal control of coronavirus (2019nCov) mathematical model; a numerical approach," Advances in Difference Equations, vol. 2020, no. 1, Article ID 528, 2020.

[8] G. Lebeau and L. Robbiano, "Contróle exact de léquation de La chaleur," Communications in Partial Differential Equations, vol. 20, no. 1-2, pp. 335-356, 1995. 
[9] B. E. Ainseba and S. Anita, "Local exact controllability of the age-dependent population dynamics with diffusion," Abstract and Applied Analysis, vol. 6, no. 6, pp. 357-368, 2001.

[10] B. E. Ainseba and M. Langlais, "On a population dynamics control problem with age dependence and spatial structure," Journal of Mathematical Analysis and Applications, vol. 248, no. 2, pp. 455-474, 2000.

[11] V. Barbu, M. Iannelli, and M. Martcheva, "On the controllability of the Lotka-McKendrick model of population dynamics," Journal of Mathematical Analysis and Applications, vol. 253, no. 1, pp. 142-165, 2001.

[12] Y. He and B. E. Ainseba, "Exact null controllability of a stage and age-structured population dynamics system," Journal of Optimization Theory and Applications, vol. 157, no. 3, pp. 918-933, 2013.

[13] Y. He and B. E. Ainseba, "Exact null controllability of the Lobesia botrana model with diffusion," Journal of Mathematical Analysis and Applications, vol. 409, no. 1, pp. 530-543, 2014.

[14] E. F. Cara, M. G. Bourgos, S. Guerrero, and J. P. Puel, "Null controllability of the heat equation with boundary Fourier conditions: the linear case," ESAIM, vol. 12, pp. 442-465, 2006.

[15] N. Hegoburu and S. Aniţa, "Null controllability via comparison results for nonlinear age-structured population dynamics," Mathematics of Control, Signals, and Systems, vol. 31, 2019.

[16] B. Ainseba and D. Picart, "Parameter identification in multistage population dynamics model," Nonlinear Analysis: Real World Applications, vol. 12, no. 6, pp. 3315-3328, 2011.

[17] B. Ainseba, D. Picart, and D. Thiéry, "An innovative multistage, physiologically structured, population model to understand the European grapevine moth dynamics," Journal of Mathematical Analysis and Applications, vol. 382, no. 1, pp. 34-46, 2011.

[18] G. F. Webb, Theory of Nonlinear Age-dependent Population Dynamics, Marcel Dekker, New York, NY, USA, 1985.

[19] A. Traoré, B. Ainseba, and O. Traoré, "On the existence of solution of a four-stage and age-structured population dynamics model," Journal of Mathematics Analysis and Applications, vol. 495, Article ID 124699, 18 pages, 2021.

[20] A. V. Fursikov and O. Yu Imanuvilov, "Controllability of evolution equations," in Lecture Notes SeriesRIM Seoul National University, Korea, East Asia, 1996.

[21] D. Chae, O. Y. Imanuvilov, and S. M. Kim, "Exact controllability for semilinear parabolic equations with Neumann boundary conditions," Journal of Dynamical and Control Systems, vol. 2, no. 4, pp. 449-483, 1996.

[22] C. Avramescu, "A fixed point theorem for multivalued mappings," Electronic Journal of Qualitative Theory of Differential Equations, vol. 17, pp. 1-10, 2004. 